\title{
A FAST AND SCALABLE METHOD FOR A-OPTIMAL DESIGN OF EXPERIMENTS FOR INFINITE-DIMENSIONAL BAYESIAN NONLINEAR INVERSE PROBLEMS
}

\author{
ALEN ALEXANDERIAN*, NOEMI PETRA ${ }^{*}$, GEORG STADLER", AND \\ OMAR GHATTAS OM $^{\dagger \ddagger}$
}

\begin{abstract}
We address the problem of optimal experimental design (OED) for Bayesian nonlinear inverse problems governed by partial differential equations (PDEs). The inverse problem seeks to infer an infinite-dimensional parameter from experimental data observed at a set of sensor locations and from the governing PDEs. The goal of the OED problem is to find an optimal placement of sensors so as to minimize the uncertainty in the inferred parameter field. Specifically, we seek an optimal subset of sensors from among a fixed set of candidate sensor locations. We formulate the OED objective function by generalizing the classical A-optimal experimental design criterion using the expected value of the trace of the posterior covariance. This expected value is computed through sample averaging over the set of likely experimental data. To cope with the infinite-dimensional character of the parameter field, we construct a Gaussian approximation to the posterior at the maximum a posteriori probability (MAP) point, and use the resulting covariance operator to define the OED objective function. We use randomized trace estimation to compute the trace of this covariance operator, which is defined only implicitly. The resulting OED problem includes as constraints the system of PDEs characterizing the MAP point, and the PDEs describing the action of the covariance (of the Gaussian approximation to the posterior) to vectors. We control the sparsity of the sensor configurations using sparsifying penalty functions. Variational adjoint methods are used to efficiently compute the gradient of the PDE-constrained OED objective function. We elaborate our OED method for the problem of determining the optimal sensor configuration to best infer the coefficient of an elliptic PDE. Furthermore, we provide numerical results for inference of the log permeability field in a porous medium flow problem. Numerical results show that the number of PDE solves required for the evaluation of the OED objective function and its gradient is essentially independent of both the parameter dimension and the sensor dimension (i.e., the number of candidate sensor locations). The number of quasi-Newton iterations for computing an OED also exhibits the same dimension invariance properties.
\end{abstract}

Key words. Optimal experimental design, A-optimal design, Bayesian inference, sensor placement, nonlinear inverse problems, randomized trace estimator, sparsified designs.

AMS subject classifications. 62K05, 35Q62, 62F15, 35R30, 35Q93, $65 \mathrm{C} 60$.

1. Introduction. We address the problem of optimal design of experiments for Bayesian nonlinear inverse problems governed by partial differential equations (PDEs). Our goal is to determine sensor locations, at which experimental data are collected, in such a way that the uncertainty in the inferred parameter field is minimized, in a sense made precise below. The numerical solution of a Bayesian inverse problem, which is just a subproblem of the optimal experimental design (OED) problem, is challenging, in particular for problems with infinite-dimensional (high-dimensional upon discretization) parameters and expensive-to-evaluate parameter-to-observable

*Institute for Computational Engineering \& Sciences, The University of Texas at Austin, Austin, TX, USA. Current address: Department of Mathematics, North Carolina State University, Raleigh, NC, USA. Email: alexanderian@ncsu.edu.

${ }^{\dagger}$ Institute for Computational Engineering \& Sciences, The University of Texas at Austin, Austin, TX, USA. omar@ices.utexas.edu.

$\ddagger$ Department of Mechanical Engineering, The University of Texas at Austin, Austin, TX, USA

$\S$ Department of Geological Sciences, The University of Texas at Austin, Austin, TX, USA

ף Applied Mathematics, School of Natural Sciences, University of California, Merced, CA, USA. Email: npetra@ucmerced.edu.

" Courant Institute of Mathematical Sciences, New York University, New York, NY, USA. Email: stadler@cims.nyu.edu. 
(forward) maps. Computing optimal experimental designs requires repeated solution of the underlying Bayesian inverse problem; hence, the OED problem inherits all of the challenges of solving the Bayesian inverse problem, which in turn inherits the computational difficulties of solving the PDEs describing the forward problem. These challenges necessitate algorithms that maximally exploit the problem structure to make OED tractable for problems that are of large scale - in the state, parameter, and data dimensions.

Related work. Standard references for OED include $[2,27,30,36]$. While most of these classical developments concern OED for inverse problems of low parameter dimension, and consider well-posed inverse problems, recently there has been an increased interest in OED for large-scale problems governed by expensive-to-solve forward models. In particular, the authors of $[9,17,19]$ present numerical methods for OED for nonlinear ill-posed inverse problems governed by large-scale models. In these papers, a frequentist point of view is taken. In particular, the OED objective function is defined as an empirical estimate of the Bayes risk of the point estimator-the solution to a Tikhonov-regularized deterministic inverse problem - for a finite-dimensional inference parameter. This amounts to solving an optimization problem for the OED that is constrained by first-order optimality conditions representing solution of an inverse problem for each member of a set of training models. There are two main differences between the work in $[17,19]$ and that proposed here. First, we address the mathematical and computational challenges stemming from the problem of OED for infinite-dimensional inverse problems. In particular, the choice of the prior, of the discretization, and of the discrete inner products is such that the discrete problems are all approximations of the same infinite-dimensional inverse problem. Second, in the OED objective, we explicitly incorporate the covariance operator of (a Gaussian approximation of) the Bayesian posterior measure, thus directly capturing the uncertainty in the inferred parameters in the objective function. This entails a more complex and difficult OED optimization problem, since now it is constrained not only by the first-order optimality conditions for the inverse problem (i.e., gradients), but also by second-order information (i.e., Hessians). Nevertheless, we demonstrate that we can construct scalable algorithms (those whose cost measured in forward PDE solves is independent of problem dimension) to solve these OED optimization problems.

Other efforts in the area include [4,23]. In [4], the authors use sequential quadratic programming (SQP) to compute optimal designs with different OED criteria for finitedimensional inverse problems governed by nonlinear systems of differential-algebraic equations (DAEs). In [23], the design of robust experiments for inverse problems governed by nonlinear DAEs is addressed; see also the review article [5]. While the inverse problems discussed in these papers are governed by nonlinear DAEs, they usually have a small to moderate number of parameters. Another idea, mainly aimed at nonlinear inverse problems with low to moderate parameter dimension, is that of $[20,21]$ in which the authors use a generalized polynomial chaos surrogate for the forward model, and utilize techniques of stochastic optimization to compute experimental designs that maximize the expected information gain as measured by the Kullback-Liebler divergence from posterior to prior. Since no closed form expression for the expected information gain is available for nonlinear Bayesian inverse problems, one must resort to computationally expensive sampling approaches. The paper [26] offers an alternate approach through a methodology based on a Laplace approximation, i.e., a Gaussian approximation, of the posterior distribution to accelerate the numerical computation of the expected information gain. 
Contributions. In this work we address the OED problem for infinite-dimensional Bayesian inverse problems, and seek scalable algorithms for its solution. We retain the infinite-dimensional structure of the problem during the development of solution methods, which not only leads to elegant mathematical formulations but also is of practical importance: studying the problem in infinite dimensions guides the choice of prior measures that are meaningful for infinite-dimensional parameters and forces one to use appropriate discretizations of the Bayesian inverse problem that avoid mesh artifacts. Moreover, the infinite-dimensional formulation provides, via the Lagrangian formalism, a straightforward way to derive adjoint-based expressions for derivatives of the OED objective. The main contributions of our work are as follows: (1) We propose a method for A-optimal experimental design for infinite-dimensional Bayesian nonlinear inverse problems; the proposed formulation aims at minimizing the expected average posterior variance. (2) We employ several approximations, which, when combined with structure-exploiting algorithms, render OED for large-scale inverse problems computationally tractable. In particular, we formulate the OED problem as a bilevel PDE-constrained optimization problem. (3) We use the problem of inferring a coefficient field in an elliptic PDE to elaborate our approach for A-optimal sensor placement. For the resulting PDE-constrained OED problem, we derive efficient adjoint-based expressions for the gradient and assess the computational complexity of the objective function evaluation and the gradient computation. (4) We present a comprehensive numerical study of the effectiveness of the OED method for optimal sensor placement for a subsurface flow inverse problem and demonstrate scalability of our framework in terms of the number of forward (and adjoint) PDE solves as the parameter and sensor dimensions increase.

Description of the method. Following an A-optimal design strategy, we seek to minimize the average posterior variance of the parameter estimates, which is given by the trace of the posterior covariance operator. For a linear inverse problem with Gaussian prior and noise distributions, a closed form expression for the posterior covariance operator is available and is independent of the experimental data [34]. For nonlinear inverse problems, however, such a closed form expression is not available and the posterior covariance operator depends on the experimental data. Since the data cannot be measured before the experiment is conducted, formally this would not lead to a meaningful OED problem. To cope with the dependence of the posterior covariance $\mathcal{C}_{\text {post }}$ on the experimental data $\mathbf{d}$, we consider the average of the trace of the posterior covariance operator over all possible experimental data:

$$
\mathrm{E}_{\mathbf{d}}\left\{\operatorname{tr}\left(\mathcal{C}_{\text {post }}(\mathbf{d})\right\},\right.
$$

where $E_{d}$ is the expectation over data. For nonlinear inverse problems, no closed form expressions for $\mathcal{C}_{\text {post }}(\mathbf{d})$ are available and the computation of $\operatorname{tr}\left(\mathcal{C}_{\text {post }}(\mathbf{d})\right)$ typically requires sampling-based methods (e.g., MCMC sampling), which are particularly expensive in high dimensions. To permit applicability to large-scale problems, we use a Gaussian approximation of the posterior measure, with mean given by the maximum a posteriori probability (MAP) point $m_{\mathrm{MAP}}=m_{\mathrm{MAP}}(\mathbf{d})$ and covariance given by the inverse of the Hessian operator $\mathcal{H}$ of the regularized data misfit functional, whose minimizer is the MAP point. This Hessian is evaluated at the MAP point, i.e., $\mathcal{H}=\mathcal{H}\left(m_{\mathrm{MAP}}(\mathbf{d}), \mathbf{d}\right)$. Notice that this approximation to the posterior is exact when the parameter-to-observable map is linear. Moreover, a Gaussian is often a good approximation to the posterior when a nonlinear parameter-to-observable map is well approximated by a linearization over the set of parameters with significant posterior 
probability. Using this Gaussian approximation, (1.1) is replaced by

$$
\mathrm{E}_{\mathbf{d}}\left\{\operatorname{tr}\left(\mathcal{H}^{-1}\left(m_{\mathrm{MAP}}(\mathbf{d}), \mathbf{d}\right)\right\} .\right.
$$

The expectation in (1.2) is approximated by averaging over a sample set $\left\{\mathbf{d}_{1}, \ldots, \mathbf{d}_{n_{\mathrm{d}}}\right\}$, where each $\mathbf{d}_{i}$ is specified according to the noise model

$$
\mathbf{d}_{i}=\boldsymbol{f}\left(m_{i}\right)+\boldsymbol{\eta}_{i},
$$

where $\boldsymbol{f}(\cdot)$ is the parameter-to-observable map, and $m_{i}$ and $\boldsymbol{\eta}_{i}$ are draws from the prior and the noise distributions, respectively. These approximations result in a formulation of the A-optimal design problem as a PDE-constrained optimization problem with constraints given by the optimality conditions of the inner optimization problem that determines the MAP point, as well as PDEs describing the application of the inverse of the Hessian.

The OED objective function involves traces of inverses of operators that are implicitly defined through solutions of PDEs. We address this difficulty by using randomized trace estimators, whose use for infinite-dimensional operators is also addressed in this paper. The experimental design is introduced in the Bayesian inverse problem through a vector of non-negative weights for possible locations where experimental data can be collected: a weight of 0 indicates absence of a sensor, and a weight of 1 means that a sensor is placed at that location. To enable use of gradient-based optimization methods for an otherwise combinatorial problem, we relax the binary assumptions on the weights and allow them to take on any value in $[0,1]$. To control the number of nonzero weights, and thus the number of sensors in the experimental design, we use a sparsifying penalty [16] that also favors binary weights [1]. Each evaluation of the OED objective requires the solution of an inner optimization problem to find the MAP point (solved using an inexact Newton-CG method), and applications of the inverse Hessian to vectors. Gradients of the OED objective with respect to the weights are computed efficiently using adjoint equations, which are derived through a Lagrangian formalism.

We elaborate the proposed OED method for the problem of inferring the log coefficient field in an elliptic PDE. Physically this can be interpreted as a subsurface flow problem in which we seek well locations at which pressure data are collected so that the uncertainty in the inferred log permeability field is minimized. We first consider a model problem in which we conduct a comprehensive numerical study of the quality of the optimal design as compared to various suboptimal designs. In these tests, we compare the designs by assessing their impact on the statistical quality of the solution of the Bayesian inverse problem. To this end, we compare the designs with respect to the average posterior variance as well as the quality of the MAP estimator which, respectively, indicate the ability of the designs to reduce uncertainty and to reconstruct "truth" log permeability fields. These tests show that optimal designs result in significant improvements over suboptimal designs with the same number of sensors. We also examine the computational complexity, in terms of the number of forward/adjoint PDE solves, of the components of our method, and numerically study its scalability. Finally, we compute an optimal experimental design for a larger-scale subsurface flow test problem with the setup and the "truth" log permeability field taken from the Society of Petroleum Engineers' 10th Comparative Solution Project (SPE10).

2. Preliminaries. In this section, we summarize the background material required for the formulation and solution of OED problems for infinite-dimensional 
Bayesian inverse problems.

2.1. Probability measures on Hilbert spaces. Let $\mathscr{H}$ denote an infinitedimensional separable real Hilbert space with inner product $\langle\cdot, \cdot\rangle_{\mathscr{H}}$ and induced norm $\|\cdot\|_{\mathscr{H}}$, and $\mathscr{B}(\mathscr{H})$ the Borel $\sigma$-algebra on $\mathscr{H}$. A probability measure on $(\mathscr{H}, \mathscr{B}(\mathscr{H}))$ is called a Borel probability measure. We consider a Borel probability measure $\mu$ on $\mathscr{H}$ with finite first and second moments with mean $\bar{m} \in \mathscr{H}$ and covariance operator $\mathcal{C}: \mathscr{H} \rightarrow \mathscr{H} . \mathcal{C}$ must be positive, self-adjoint, and of trace-class [10] and satisfies

$$
\int_{\mathscr{H}}\|m-\bar{m}\|_{\mathscr{H}}^{2} \mu(d m)=\operatorname{tr}(\mathcal{C}) .
$$

A Borel probability measure $\mu$ on $\mathscr{H}$ is said to be Gaussian if and only if for each $x \in \mathscr{H}$, the functional $u \mapsto\langle x, u\rangle_{\mathscr{H}} \in \mathbb{R}$, viewed as a real-valued random variable on $(\mathscr{H}, \mathscr{B}(\mathscr{H}), \mu)$, is Gaussian $[10,29]$. We denote by $\mathcal{N}(\bar{m}, \mathcal{C})$ a Gaussian measure on $\mathscr{H}$ with mean $\bar{m}$ and covariance operator $\mathcal{C}$.

In the present work, $\mathscr{H}=L^{2}(\mathcal{D})$ with the standard $L^{2}$-inner product $\langle\cdot, \cdot\rangle$ and induced norm $\|\cdot\|$, where $\mathcal{D} \subset \mathbb{R}^{d}(d=2,3)$ is a bounded domain with sufficiently regular boundary. Let $(\Omega, \Sigma, \mathrm{P})$ be a probability space and let $m:(\Omega, \Sigma, \mathrm{P}) \rightarrow(\mathscr{H}, \mathscr{B}(\mathscr{H}))$ be an $\mathscr{H}$-valued random variable with law $\mu$, i.e., $\mu(E)=\mathrm{P}(m \in E)$, for $E \in \mathscr{B}(\mathscr{H})$. Notice that for each $\omega \in \Omega, m(\cdot, \omega): \mathcal{D} \rightarrow \mathbb{R}$ is a function. Alternatively, we may consider $m$ as real-valued function defined on $\mathcal{D} \times \Omega$, where for each $\boldsymbol{x} \in \mathcal{D}, m(\boldsymbol{x}, \cdot)$ is a real-valued random variable, i.e., $m$ is a random field. In this paper, we consider random fields that are jointly measurable on $(\mathscr{H}, \mathscr{B}(\mathscr{H})) \otimes(\Omega, \Sigma)$ and have finite second moment. Invoking Tonelli's theorem, the pointwise variance $\operatorname{var}\{m(\boldsymbol{x})\}, \boldsymbol{x} \in \mathcal{D}$, satisfies,

$$
\begin{aligned}
\int_{\mathcal{D}} \operatorname{var}\{m(\boldsymbol{x})\} d \boldsymbol{x} & =\int_{\mathcal{D}} \int_{\Omega}(m(\boldsymbol{x}, \omega)-\bar{m}(\boldsymbol{x}))^{2} \mathrm{P}(d \omega) d \boldsymbol{x} \\
& =\int_{\Omega} \int_{\mathcal{D}}(m(\boldsymbol{x}, \omega)-\bar{m}(\boldsymbol{x}))^{2} d \boldsymbol{x} \mathrm{P}(d \omega) \\
& =\int_{\Omega}\|m(\cdot, \omega)-\bar{m}(\cdot)\|^{2} \mathrm{P}(d \omega) \\
& =\int_{\mathscr{H}}\|m-\bar{m}\|^{2} \mu(d m)=\operatorname{tr}(\mathcal{C}),
\end{aligned}
$$

where as before $\bar{m}$ denotes the mean of $m$. This shows that the trace of the covariance operator is proportional to the average of the pointwise variance over the physical domain $\mathcal{D}$ - a relation that is central to our formulation of A-optimal experimental design in an infinite-dimensional Hilbert space.

2.2. Bayesian inversion in an infinite-dimensional Hilbert space. We consider the problem of inferring the law of the parameter $m$, modeled as an $\mathscr{H}$ valued random variable, from observations. Here, we describe the main ingredients of a Bayesian inverse problem.

The prior distribution law. We use a Gaussian prior distribution law $\mu_{\mathrm{pr}}=$ $\mathcal{N}\left(m_{\mathrm{pr}}, \mathcal{C}_{\mathrm{pr}}\right)$ for the inference parameter, where the prior mean $m_{\mathrm{pr}}$ is a sufficiently regular element of $\mathscr{H}$ and $\mathcal{C}_{\text {pr }}: \mathscr{H} \rightarrow \mathscr{H}$ a strictly positive self-adjoint traceclass operator given by the inverse of a differential operator. To be precise, following [7,33], we use $\mathcal{C}=\mathcal{A}^{-2}$, where $\mathcal{A}$ is a Laplacian-like operator; this choice ensures that in two and three space dimensions, $\mathcal{C}$ is a trace-class operator and, thus, 
the distribution is well-defined. The measure $\mu_{\mathrm{pr}}$ induces the Cameron-Martin space $\mathscr{E}=\operatorname{range}\left(\mathcal{C}_{\text {pr }}^{1 / 2}\right)=\operatorname{dom}(\mathcal{A})$ which is a dense subspace of $\mathscr{H}$ and is endowed with the inner product,

$$
\langle x, y\rangle_{\mathscr{E}}=\langle\mathcal{A} x, \mathcal{A} y\rangle, \quad x, y \in \mathscr{E}
$$

In what follows, we assume that the prior mean $m_{\text {pr }}$ is an element of $\mathscr{E}$.

Note that the choice of a prior that is meaningful in a function space setting is a known challenge and an active field of research, $[11,13,24,33]$. Gaussian priors are a common choice for infinite-dimensional Bayesian inverse problems. From a practical point of view, the use of a Gaussian prior is a modeling choice. The prior mean describes our best guess about the uncertain parameter, which could be obtained from existing measurements or from other available information. The covariance operator allows modeling of the correlation lengths and of the pointwise variance. The choices for mean and prior might depend on the properties that are relevant for the parameter-to-observable map. For instance, for the subsurface flow problems considered in sections 6 and 7, the pore-scale rock features only influence the flow in an averaged sense. Thus, considering smoother permeability fields that describe different types of rocks is sufficient and an effective permeability field is all one can hope to infer from observations. For the prior defined above, the Green's function of the differential operator $\mathcal{A}$ describes the correlation between the parameter values at different spational points, and so one can choose $\mathcal{A}$ such that it incorporates the desired correlation information. We also mention the article [25], where a detailed study of this relation between explicitly specified Matérn-type Gaussian random fields and PDE operators is presented.

The parameter-to-observable map and the data likelihood. Next, we introduce the data likelihood, which describes the distribution of experimental data $\mathbf{d}$ for a given parameter $m \in \mathscr{H}$. Here, we consider finite-dimensional observations $\mathbf{d} \in \mathbb{R}^{q}$, and denote by $\pi_{\text {like }}(\mathbf{d} \mid m)$ the likelihood probability density function (pdf). Let $\boldsymbol{f}: \mathscr{H} \rightarrow$ $\mathbb{R}^{q}$ denote a parameter-to-observable map, which is a sufficiently regular (see [33]) deterministic function that maps a parameter $m \in \mathscr{H}$ to an experimental data d. In the problems we target, an evaluation of $\boldsymbol{f}(m)$ typically requires a forward solve (typically a PDE solve) followed by the application of an observation operator. We consider an additive Gaussian noise model

$$
\mathbf{d}=\boldsymbol{f}(m)+\boldsymbol{\eta}, \quad \boldsymbol{\eta} \sim \mathcal{N}\left(\mathbf{0}, \boldsymbol{\Gamma}_{\text {noise }}\right)
$$

where $\boldsymbol{\Gamma}_{\text {noise }} \in \mathbb{R}^{q \times q}$ is the noise covariance matrix. Note that $\boldsymbol{\eta}$ is independent of $m$ and thus $\mathbf{d} \mid m \sim \mathcal{N}\left(\boldsymbol{f}(m), \boldsymbol{\Gamma}_{\text {noise }}\right)$ and the likelihood is given by

$$
\pi_{\text {like }}(\mathbf{d} \mid m) \propto \exp \left\{-\frac{1}{2}(\boldsymbol{f}(m)-\mathbf{d})^{T} \boldsymbol{\Gamma}_{\text {noise }}^{-1}(\boldsymbol{f}(m)-\mathbf{d})\right\} .
$$

The Bayes formula in infinite dimensions. The solution of a Bayesian inverse problem is the posterior measure, which describes the probability law of the parameter $m$ conditioned on observed data $\mathbf{d}$. The relationship between the prior measure, the data likelihood, and this posterior measure is described by the Bayes formula, which in the infinite-dimensional Hilbert space settings is given by [33],

$$
\frac{d \mu_{\text {post }}^{\mathbf{d}}}{d \mu_{\mathrm{pr}}} \propto \pi_{\text {like }}(\mathbf{d} \mid m) \text {. }
$$


Here, the left hand side is the Radon-Nikodym derivative [37] of the posterior probability measure $\mu_{\text {post }}^{\mathrm{d}}$ with respect to the prior measure $\mu_{\mathrm{pr}}$. See [33] for conditions on the parameter-to-observable map $f$ that ensure that the above Bayes formula holds.

2.3. The maximum a posteriori probability (MAP) point. For a finitedimensional inference problem, the MAP point is a point in the parameter space at which the posterior pdf is maximized. While this notion does not extend directly to infinite dimensions, one can define the MAP point $m_{\mathrm{MAP}}$ as the point $m \in \mathscr{H}$ that maximizes the posterior probability of balls of radius $\varepsilon$ centered at $m$, as $\varepsilon \rightarrow 0$. Analogous to the finite-dimensional case, the MAP point can be found by minimizing the functional $\mathcal{J}: \mathscr{E} \rightarrow \mathbb{R}$ given by [12],

$$
\mathcal{J}(m):=\frac{1}{2}\left\langle\boldsymbol{f}(m)-\mathbf{d}, \boldsymbol{\Gamma}_{\text {noise }}^{-1}(\boldsymbol{f}(m)-\mathbf{d})\right\rangle_{\mathbb{R}^{q}}+\frac{1}{2}\left\langle m-m_{\mathrm{pr}}, m-m_{\mathrm{pr}}\right\rangle_{\mathscr{E}} .
$$

That is,

$$
m_{\mathrm{MAP}}=\underset{m \in \mathscr{E}}{\arg \min } \mathcal{J}(m) .
$$

The existence of solutions to the above optimization problem follows standard arguments [33]. We point out that (2.2) is equivalent to a deterministic inverse problem, where inner products in the regularized data misfit functional $\mathcal{J}$ are weighted according to the statistical description of the problem, i.e., with the noise and prior covariance operators. Note that the MAP point $m_{\mathrm{MAP}}$ depends on the experimental data $\mathbf{d}$. This is a challenge in the context of OED, where data are not available a priori. Moreover, the solution of (2.2) is not guaranteed to be unique.

2.4. Experimental design in a Bayesian inverse problem. Next, we define what we mean by an experimental design, and describe how an experimental design enters in the Bayesian inverse problem formulation. We consider the problem of optimal placement of sensors that measure experimental data. We fix a collection of candidate sensor locations, $\boldsymbol{x}_{1}, \ldots, \boldsymbol{x}_{n_{s}}$ in $\mathcal{D}$ and assign to each location a non-negative weight $w_{i}$, which controls whether experimental data are gathered at location $\boldsymbol{x}_{i}$, for $i=1, \ldots, n_{s}$. Thus, a design is fully specified by a weight vector $\boldsymbol{w}:=\left(w_{1}, \ldots, w_{n_{s}}\right) \in$ $\mathbb{R}_{\geq 0}^{n_{s}}$. Since an experimental design determines the subset of the set of candidate sensor locations at which data are collected, $\boldsymbol{w}$ enters the Bayesian inverse problem through the data likelihood, amounting to a weighted data likelihood:

$$
\pi_{\text {like }}(\mathbf{d} \mid m ; \boldsymbol{w}) \propto \exp \left\{-\frac{1}{2}(\boldsymbol{f}(m)-\mathbf{d})^{T} \mathbf{W}^{1 / 2} \boldsymbol{\Gamma}_{\text {noise }}^{-1} \mathbf{W}^{1 / 2}(\boldsymbol{f}(m)-\mathbf{d})\right\},
$$

where $\mathbf{W}=\operatorname{diag}\left(w_{1}, \ldots, w_{n_{s}}\right)$. Notice that this formulation assumes that the dimension of the data vector equals the number of candidate sensor locations, i.e., $q=n_{s}$.

Here, we consider uncorrelated observations, that is, the noise covariance is diagonal, $\boldsymbol{\Gamma}_{\text {noise }}=\operatorname{diag}\left(\sigma_{1}^{2}, \ldots, \sigma_{n_{s}}^{2}\right)$. Thus,

$$
\mathbf{W}_{\sigma}:=\mathbf{W}^{1 / 2} \boldsymbol{\Gamma}_{\text {noise }}^{-1} \mathbf{W}^{1 / 2}=\operatorname{diag}\left(w_{1} / \sigma_{1}^{2}, \ldots, w_{n_{s}} / \sigma_{n_{s}}^{2}\right) .
$$

The solution of the Bayesian inverse problem with the weighted likelihood (2.3) now additionally depends on the design $\boldsymbol{w}$. For example, the MAP point (or estimator) $m_{\mathrm{MAP}}$ is the minimizer, with respect to $m$, of the weighted cost functional,

$$
\mathcal{J}(m, \boldsymbol{w} ; \mathbf{d}):=\frac{1}{2}\left\langle\boldsymbol{f}(m)-\mathbf{d}, \mathbf{W}_{\sigma}(\boldsymbol{f}(m)-\mathbf{d})\right\rangle_{\mathbb{R}^{q}}+\frac{1}{2}\left\langle m-m_{\mathrm{pr}}, m-m_{\mathrm{pr}}\right\rangle_{\mathscr{E}},
$$


i.e.,

$$
m_{\mathrm{MAP}}(\boldsymbol{w} ; \mathbf{d})=\underset{m \in \mathscr{E}}{\arg \min } \mathcal{J}(m, \boldsymbol{w} ; \mathbf{d}) .
$$

Other statistics of the posterior, such as the mean and the covariance operator, also depend on $\boldsymbol{w}$.

In classical OED formulations $[2,27,30,36]$, one commonly interprets the components of a design vector $\boldsymbol{w}$ as probability masses for candidate sensor location, i.e., $w_{i} \geq 0$ and $\sum w_{i}=1$. A practitioner might place sensors at the candidate locations whose weights are large or use the weights to decide which experiments to perform, and how often to perform them (if experiments can be repeated) to reduce the experimental noise level through repeated experiments. An alternate point of view is to neglect the constraint $\sum w_{i}=1$ and to incorporate a penalty function $P(\boldsymbol{w})$ instead, which associates a cost to each sensor placed $[1,16,18]$. The simplest-to-interpret weight vector $\boldsymbol{w}$ contains 0 's where no sensor is placed and 1's in locations where sensors are placed. This leads to a binary optimization problem, which can be challenging to solve. Thus, we relax the binary assumption on the components of the weight vector, and allow the weights to take values in the interval $[0,1]$ and enforce binary weights through properly chosen sparsifying penalty functions, or continuation with a family of penalty functions (see section 5.6).

2.5. Randomized trace estimation. We address A-optimal experimental design problems, which require minimization of traces of large dense covariance matrices that are defined implicitly through their applications to vectors. In our OED method, we approximate traces of covariance matrices using randomized trace estimators. These estimators approximate the trace of a matrix $\mathbf{A} \in \mathbb{R}^{n \times n}$ via Monte-Carlo estimates of the form $\operatorname{tr}(\mathbf{A}) \approx \frac{1}{n_{\mathrm{tr}}} \sum_{k=1}^{n_{\mathrm{tr}}}\left\langle\boldsymbol{z}_{k}, \mathbf{A} \boldsymbol{z}_{k}\right\rangle_{\mathbb{R}^{n}}$, where the vectors $\boldsymbol{z}_{k}$ are random $n$-vectors. Reasonably accurate estimation of traces of high-dimensional covariance matrices are possible with a small number of random vectors; see e.g., [3,32] for descriptions of different trace estimators and their convergence properties, and $[1,16,18]$ for discussions regarding the use of randomized trace estimators for high-dimensional implicitly defined covariance operators. There are several possibilities for the choice of random vectors $\boldsymbol{z}_{k}$. The Hutchinson estimator [22] uses random vectors with \pm 1 entries, each with a probability of $1 / 2$. Another possibility, used in this paper, is the Gaussian trace estimator, which uses Gaussian random vectors with independent standard normal entries.

In our numerical computations, we estimate traces of matrices that are discretizations of covariance operators defined on an infinite-dimensional Hilbert space. Thus, we next briefly justify randomized trace estimation in infinite dimensions. In particular, to define the infinite-dimensional analog of the Gaussian trace estimator, we consider an $\mathscr{H}$-valued random variable $Z_{\delta}$ whose law is given by $\mu_{\delta}=\mathcal{N}\left(0, \mathcal{C}_{\delta}\right)$, where $\mathcal{C}_{\delta}=(-\delta \Delta+I)^{-2}$; here, $\Delta$ denotes the Laplacian operator with homogeneous Neumann boundary conditions, and $\delta$ is a positive real number. Note that $\mathcal{C}_{\delta}$ so constructed is positive, self-adjoint, and of trace-class on $L^{2}(\mathcal{D})$, with $\mathcal{D} \subseteq \mathbb{R}^{d}, d=2,3$. Let $\mathcal{A}$ be a positive self-adjoint trace-class operator on $\mathscr{H}$. First, note that

$$
\mathrm{E}\left\{\left\langle Z_{\delta}, \mathcal{A} Z_{\delta}\right\rangle\right\}=\int_{\mathscr{H}}\langle z, \mathcal{A} z\rangle \mu_{\delta}(d z)=\operatorname{tr}\left(\mathcal{A C}_{\delta}\right) .
$$

Moreover, as shown in Appendix $\mathrm{A}, \operatorname{tr}(\mathcal{A})=\lim _{\delta \rightarrow 0} \operatorname{tr}\left(\mathcal{A C}_{\delta}\right)$. Hence, choosing small values of $\delta$ provides reasonable estimates for $\operatorname{tr}(\mathcal{A})$. Therefore, one is justified to use 
Monte Carlo estimates of the form,

$$
\operatorname{tr}(\mathcal{A}) \approx \frac{1}{n_{\mathrm{tr}}} \sum_{i=1}^{n_{\mathrm{tr}}}\left\langle z_{i}, \mathcal{A} z_{i}\right\rangle
$$

where $z_{i}$ are realizations of $Z_{\delta}$ for a sufficiently small $\delta$ (in the finite-dimensional case, we can take $\delta=0)$.

3. A-optimal design for Bayesian linear inverse problems. The classical definition of an A-optimal design is for inverse problems where the parameter-toobservable map $\boldsymbol{f}$ is linear and one assumes an additive Gaussian noise model. In this case, the posterior covariance operator does not depend on the experimental data. Denoting by $\mathcal{C}_{\text {post }}(\boldsymbol{w})$ the covariance operator of the posterior measure $\mu_{\text {post }}^{\mathrm{d}}$ for a given design vector $\boldsymbol{w}$, an A-optimal design is one that minimizes the average posterior variance. This is equivalent to minimizing $\operatorname{tr}\left(\mathcal{C}_{\text {post }}(\boldsymbol{w})\right)$. Denoting the linear parameter-to-observable map by $\mathbf{F}: \mathscr{H} \rightarrow \mathbb{R}^{q}$ and assuming a Gaussian prior $\mu_{\mathrm{pr}}=$ $\mathcal{N}\left(\cdot, \mathcal{C}_{\mathrm{pr}}\right)$, the posterior covariance operator is $\mathcal{C}_{\text {post }}(\boldsymbol{w})=\left(\mathbf{F}^{*} \mathbf{W}_{\sigma} \mathbf{F}+\mathcal{C}_{\mathrm{pr}}^{-1}\right)^{-1}$, with $\mathbf{W}_{\sigma}$ as in (2.4). Notice that $\mathbf{F}$ is independent of the parameter $m$ and the experimental data d. Using a low rank singular value decomposition of the prior-preconditioned parameter-to-observable map $\mathbf{F} \mathcal{C}_{\mathrm{pr}}^{1 / 2}$, computed once upfront, enables evaluating the A-optimal objective function and its gradient without further PDE solves; see $[1,18]$.

This A-optimal design approach leads to the following optimization problem:

$$
\min _{\boldsymbol{w} \in[0,1]^{n_{s}}} \operatorname{tr}\left(\mathcal{C}_{\text {post }}(\boldsymbol{w})\right)+\gamma P(\boldsymbol{w}),
$$

where $\gamma P(\boldsymbol{w})$ controls the sparsity of the design $\boldsymbol{w}$. There are various options for choosing a sparsifying penalty function $P(\boldsymbol{w})$. One possibility is to use $P(\boldsymbol{w})=\sum_{i} w_{i}$, which amounts to an $\ell^{1}$ penalty. Here, we use a continuation strategy with a sequence of penalty functions that asymptotically approximate the $\ell^{0}$ - "norm"; see section 5.6 and [1].

4. A-optimal design for Bayesian nonlinear inverse problems. In this section, we present a formulation of the A-optimal experimental design criterion for infinite-dimensional Bayesian nonlinear inverse problems. To make the resulting OED problem computationally tractable, we introduce a series of approximations, such that the formulation culminates in a Hessian constrained bilevel optimization problem.

4.1. Formulation. For a design vector $\boldsymbol{w}$ and experimental data $\mathbf{d}$, the Bayesian inverse problem with the weighted data likelihood (2.3) is given by

$$
\frac{d \mu_{\mathrm{post}}^{\mathbf{d}}}{d \mu_{\mathrm{pr}}} \propto \pi_{\text {like }}(\mathbf{d} \mid m ; \boldsymbol{w}) .
$$

Following an A-optimal design criterion, we seek to minimize the average posterior variance of the inferred parameter over all possible design vectors $\boldsymbol{w}$. From (2.1) it follows that the average variance is given by $\operatorname{tr}\left[\mathcal{C}_{\text {post }}(\boldsymbol{w} ; \mathbf{d})\right]$, where $\mathcal{C}_{\text {post }}$ is the covariance operator corresponding to the posterior measure. Note that for a fixed experimental design vector $\boldsymbol{w}$, the result of the inference still depends on the experimental data $\mathbf{d}$. Since experimental data is, in general, not available a priori, we average $\operatorname{tr}\left[\mathcal{C}_{\text {post }}(\boldsymbol{w} ; \mathbf{d})\right]$ over the experimental data d, which, for given $m \in \mathscr{H}$, are distributed according to $\mathcal{N}\left(\boldsymbol{f}(m), \boldsymbol{\Gamma}_{\text {noise }}\right)$, as specified by the data likelihood. Notice that this distribution of $\mathbf{d}$ 
is conditioned on $m$, the parameter in the Bayesian inverse problem. To address this issue, we rely on our prior knowledge of the parameter $m$ as described by the prior measure, and define the expected average posterior variance $\Psi$ as follows:

$$
\Psi(\boldsymbol{w}):=\mathrm{E}_{\mu_{\mathrm{pr}}} \mathrm{E}_{\mathbf{d} \mid m}\left\{\operatorname{tr}\left[\mathcal{C}_{\text {post }}(\boldsymbol{w} ; \mathbf{d})\right]\right\}=\int_{\mathscr{H}} \int_{\mathbb{R}^{q}} \operatorname{tr}\left[\mathcal{C}_{\text {post }}(\boldsymbol{w} ; \mathbf{d})\right] \mu_{\mathbf{d} \mid m}(d \mathbf{d}) \mu_{\mathrm{pr}}(d m),
$$

where $\mu_{\mathbf{d} \mid m}=\mathcal{N}\left(\boldsymbol{f}(m), \boldsymbol{\Gamma}_{\text {noise }}\right)$.

4.2. Gaussian approximation of the posterior measure. If the parameterto-observable map $\boldsymbol{f}$ is linear, and given a Gaussian prior distribution and an additive Gaussian noise model, the posterior is also Gaussian, with mean and covariance given by closed form expressions, namely the MAP point and the inverse of the Hessian of the functional $\mathcal{J}$ defined in (2.5), respectively, [33,34]. However, if $\boldsymbol{f}$ is nonlinear, the posterior is not Gaussian and there exists no closed-form expression for the posterior covariance operator. As a consequence, one has to rely on techniques such as Markov chain Monte Carlo sampling to compute the average posterior variance [31]. This requires a large number of statistically independent samples, which in turn requires many evaluations of the parameter-to-observable map $\boldsymbol{f}$, which can make sampling computationally extremely expensive, in particular for high-dimensional problems and expensive-to-evaluate parameter-to-observable maps $\boldsymbol{f}$. Thus, to make the problem at hand tractable, we consider a Gaussian approximation of the posterior measure at the MAP point. That is, given an experimental design $\boldsymbol{w}$ and a realization of the data $\mathbf{d}$, we compute the MAP point $m_{\mathrm{MAP}}=m_{\mathrm{MAP}}(\boldsymbol{w} ; \mathbf{d})$ and define the Gaussian approximation of $\mu_{\text {post }}^{\mathbf{d}}$ as

$$
\mu_{\text {post }}^{\mathbf{d}, \mathrm{G}}:=\mathcal{N}\left(m_{\mathrm{MAP}}(\boldsymbol{w} ; \mathbf{d}), \mathcal{H}^{-1}\left(m_{\mathrm{MAP}}(\boldsymbol{w} ; \mathbf{d}), \boldsymbol{w} ; \mathbf{d}\right)\right),
$$

where $\mathcal{H}\left(m_{\mathrm{MAP}}(\boldsymbol{w} ; \mathbf{d}), \boldsymbol{w} ; \mathbf{d}\right)$ is the Hessian of $(2.5)$ (or an approximation of the Hessian, e.g., the Gauss-Newton approximation). Note that, in general, $\mathcal{H}$ depends on the design $\boldsymbol{w}$ and data $\mathbf{d}$ both explicitly, and implicitly through the MAP point. Using this Gaussian approximation, we proceed to define the following approximation $\Psi^{\mathrm{G}}$ for the OED objective function $\Psi$ defined in (4.1):

$$
\Psi^{\mathrm{G}}(\boldsymbol{w})=\mathrm{E}_{\mu_{\mathrm{pr}}} \mathrm{E}_{\mathbf{d} \mid m}\left\{\operatorname{tr}\left[\mathcal{H}^{-1}\left(m_{\mathrm{MAP}}(\boldsymbol{w} ; \mathbf{d}), \boldsymbol{w} ; \mathbf{d}\right)\right]\right\} .
$$

To ensure that the Gaussian approximation $\mu_{\text {post }}^{\mathrm{d}, \mathrm{G}}$ is well defined, we make the following assumption:

Assumption 4.1. For every experimental data $\mathbf{d}$ and every design vector $\boldsymbol{w}$ from the admissible set of designs the inverse of the Hessian $\mathcal{H}^{-1}\left(m_{M A P}(\boldsymbol{w} ; \mathbf{d}), \boldsymbol{w} ; \mathbf{d}\right)$ exists and is a positive trace-class operator.

4.3. Sample averaging and randomized trace estimation. The evaluation of $\Psi^{\mathrm{G}}$ given in (4.2) involves integration over an infinite-dimensional (upon discretization, high-dimensional) space. To approximate this integration, we replace $\Psi^{\mathrm{G}}$ by the Monte Carlo sum

$$
\Psi_{n_{\mathrm{d}}}^{\mathrm{G}}(\boldsymbol{w})=\frac{1}{n_{\mathrm{d}}} \sum_{i=1}^{n_{\mathrm{d}}} \operatorname{tr}\left[\mathcal{H}^{-1}\left(m_{\mathrm{MAP}}\left(\boldsymbol{w} ; \mathbf{d}_{i}\right), \boldsymbol{w} ; \mathbf{d}_{i}\right)\right] .
$$

The data samples $\mathbf{d}_{i}$ are given by $\mathbf{d}_{i}=\boldsymbol{f}\left(m_{i}\right)+\boldsymbol{\eta}_{i}$, where $\left\{\left(m_{i}, \boldsymbol{\eta}_{i}\right)\right\}_{i=1}^{n_{\mathrm{d}}}$ is a sample set from the product space $\left(\mathscr{H}, \mu_{\mathrm{pr}}\right) \times\left(\mathbb{R}^{q}, \mathcal{N}\left(\mathbf{0}, \boldsymbol{\Gamma}_{\text {noise }}\right)\right)$. Note that in practical computations usually only a moderate number of data samples can be afforded for reasons 
that will become clear later in the paper. From a frequentist's perspective, the draws $m_{i}$ from the prior can be considered as training models. Note that the draws $\mathbf{d}_{i}$ enter in (4.3) through the MAP point and the Hessian at the MAP point. This incorporates the physical properties of the parameter-to-observable map $f$ in the OED objective function. For instance, if $\boldsymbol{f}$ damps highly oscillatory modes of the parameters, $\Psi^{\mathrm{G}}$ is insensitive to the highly oscillatory modes of $m_{i}$ used to compute $\mathbf{d}_{i}$. This indirect dependence of the OED objective on "training" draws from the prior is in contrast to the OED approach for nonlinear inverse problems proposed in $[17,19]$, in which training models enter in the OED objective function directly.

The objective function (4.3) involves the trace of $\mathcal{H}_{i}^{-1}=\mathcal{H}^{-1}\left(m_{\mathrm{MAP}}\left(\boldsymbol{w} ; \mathbf{d}_{i}\right), \boldsymbol{w} ; \mathbf{d}_{i}\right)$. This trace is given by $\operatorname{tr}\left[\mathcal{H}_{i}^{-1}\right]=\sum_{k=1}^{\infty}\left\langle e_{k}, \mathcal{H}_{i}^{-1} e_{k}\right\rangle$, where $\left\{e_{k}\right\}$ is a complete orthonormal set in $\mathscr{H}$. Thus, we can write (4.3) as follows:

$$
\Psi_{n_{\mathrm{d}}}^{\mathrm{G}}(\boldsymbol{w})=\frac{1}{n_{\mathrm{d}}} \sum_{i=1}^{n_{\mathrm{d}}} \sum_{k=1}^{\infty}\left\langle e_{k}, y_{i k}\right\rangle,
$$

where for $i \in\left\{1, \ldots, n_{\mathrm{d}}\right\}$ and $k \in \mathbb{N}$ :

$$
\begin{aligned}
m_{\mathrm{MAP}}\left(\boldsymbol{w} ; \mathbf{d}_{i}\right) & =\underset{m}{\arg \min } \mathcal{J}\left(m, \boldsymbol{w} ; \mathbf{d}_{i}\right) \\
\mathcal{H}\left(m_{\mathrm{MAP}}\left(\boldsymbol{w} ; \mathbf{d}_{i}\right), \boldsymbol{w} ; \mathbf{d}_{i}\right) y_{i k} & =e_{k} .
\end{aligned}
$$

Notice that for each $i \in\left\{1, \ldots, n_{s}\right\}$, we obtain a MAP point $m_{\mathrm{MAP}}\left(\boldsymbol{w} ; \mathbf{d}_{i}\right)$, which is used to define the corresponding Hessian operator $\mathcal{H}_{i}=\mathcal{H}\left(m_{\mathrm{MAP}}\left(\boldsymbol{w} ; \mathbf{d}_{i}\right), \boldsymbol{w} ; \mathbf{d}_{i}\right)$.

The computation of the trace based on a complete orthogonal basis as in (4.4) is not practical. We thus use a randomized trace estimator (see section 2.5) to obtain an expression that can be computed efficiently. This final approximation step results in a computationally tractable OED objective function, which is used in the formulation of an A-optimal experimental design problem below.

4.4. The resulting A-optimal experimental design problem. The definitions and approximations discussed above result in the following formulation of an A-optimal design objective function for a nonlinear Bayesian inverse problem:

$$
\hat{\Psi}(\boldsymbol{w}):=\frac{1}{n_{\mathrm{d}} n_{\mathrm{tr}}} \sum_{i=1}^{n_{\mathrm{d}}} \sum_{k=1}^{n_{\mathrm{tr}}}\left\langle z_{k}, y_{i k}\right\rangle,
$$

where $z_{k}, k \in\left\{1, \ldots, n_{\mathrm{tr}}\right\}$, are random vectors as discussed in section 2.5, and for $i \in\left\{1, \ldots, n_{\mathrm{d}}\right\}, y_{i k}$ is defined through

$$
\begin{aligned}
m_{\mathrm{MAP}}\left(\boldsymbol{w} ; \mathbf{d}_{i}\right) & =\underset{m}{\arg \min } \mathcal{J}\left(m, \boldsymbol{w} ; \mathbf{d}_{i}\right), \\
\mathcal{H}\left(m_{\mathrm{MAP}}\left(\boldsymbol{w} ; \mathbf{d}_{i}\right), \boldsymbol{w} ; \mathbf{d}_{i}\right) y_{i k} & =z_{k} .
\end{aligned}
$$

The corresponding A-optimal experimental design optimization problem, with a sparsifying penalty term (as discussed in section 2.4 ) is given by

$$
\min _{\boldsymbol{w} \in[0,1]^{n_{s}}} \hat{\Psi}(\boldsymbol{w})+\gamma P(\boldsymbol{w}) .
$$

Since we rely on gradient-based methods to solve $(\mathcal{P})$, in addition to Assumption 4.1, we require the following assumption to hold.

Assumption 4.2. The OED objective $\hat{\Psi}(\cdot)$ is continuously differentiable with respect to the weight vector $\boldsymbol{w}$ for all $\boldsymbol{w} \in[0,1]^{n_{s}}$. 
5. OED for coefficient field inference in an elliptic PDE. Next, we elaborate our approach for A-optimal design of experiments to the inference of the log coefficient field in an elliptic partial differential equation, i.e., we consider the forward model,

$$
\begin{aligned}
-\nabla \cdot\left(e^{m} \nabla u\right) & =f & & \text { in } \mathcal{D}, \\
u & =g & & \text { on } \Gamma_{D}, \\
e^{m} \nabla u \cdot \boldsymbol{n} & =h & & \text { on } \Gamma_{N},
\end{aligned}
$$

where $\mathcal{D} \subset \mathbb{R}^{d}(d=2,3)$ is an open bounded domain with sufficiently smooth boundary $\Gamma=\Gamma_{D} \cup \Gamma_{N}, \Gamma_{D} \cap \Gamma_{N}=\emptyset$. Here, $u$ is the state variable, $f \in L^{2}(\mathcal{D})$ is a source term, and $g \in H^{1 / 2}\left(\Gamma_{D}\right)$ and $h \in L^{2}\left(\Gamma_{N}\right)$ are Dirichlet and Neumann boundary data, respectively. The prior distribution for $m$ ensures that, almost surely, realizations of $m$ are continuous in $\overline{\mathcal{D}}$. Hence, $e^{m}$ is positive and bounded, ensuring existence of a solution of (5.1). Define the spaces,

$$
\mathscr{V}_{g}=\left\{v \in H^{1}(\mathcal{D}):\left.v\right|_{\Gamma_{D}}=g\right\}, \quad \mathscr{V}_{0}=\left\{v \in H^{1}(\mathcal{D}):\left.v\right|_{\Gamma_{D}}=0\right\},
$$

where $H^{1}(\mathcal{D})$ is the Sobolev space of functions in $L^{2}(\mathcal{D})$ with square integrable derivatives. Then, the weak form of (5.1) reads as follows: Find $u \in \mathscr{V}_{g}$ such that

$$
\left\langle e^{m} \nabla u, \nabla p\right\rangle=\langle f, p\rangle+\langle h, p\rangle_{\Gamma_{N}}, \quad \forall p \in \mathscr{V}_{0} .
$$

In the following subsections, we specialize the OED problem $(\mathcal{P})$ for the inference of $m$ in (5.1) from pointwise observations of the state variable $u$. For theoretical aspects of the Bayesian approach to estimating the coefficient field in elliptic PDEs we refer to $[13,33]$.

In sections 5.1 and 5.2, we derive expressions for the first and second derivatives of the "inner" problem, i.e., the inverse problem whose solution is the MAP point. In Section 5.3 we formulate the OED problem as a bilevel optimization problem, constrained by PDEs characterizing the MAP point and PDEs defining the action of the inverse Hessian. Then, in section 5.4, we formulate the OED objective resulting in the "outer" OED optimization problem, and derive expressions for the gradient of the OED objective using associated adjoint equations. A discussion of the complexity of evaluating the OED objective and its gradient, in terms of the number of forward PDE solves, is provided in section 5.5.

5.1. Optimality system for the MAP point. We first specialize the (weighted) cost functional (2.5), whose minimizer is the MAP point, for the problem of inferring $m$ in (5.1) from observations $\mathcal{B} u$, where $\mathcal{B}$ is a linear observation operator that extracts measurements from $u$ :

$$
\mathcal{J}(m, \boldsymbol{w} ; \mathbf{d})=\frac{1}{2}\left\langle\mathcal{B} u-\mathbf{d}, \mathbf{W}_{\sigma}(\mathcal{B} u-\mathbf{d})\right\rangle_{\mathbb{R}^{q}}+\frac{1}{2}\left\langle m-m_{\mathrm{pr}}, m-m_{\mathrm{pr}}\right\rangle_{\mathscr{E}} .
$$

Here, for a given $m$, the state variable $u$ is the solution to (5.1), $m_{\mathrm{pr}}$ is the prior mean of the $\log$ coefficient field, and $\mathbf{d} \in \mathbb{R}^{q}$ is a given data vector. Note that every evaluation of the OED objective function in $(\mathcal{P})$ with a given design $\boldsymbol{w}$ requires minimization of the PDE-constrained data misfit cost functional in (5.2). Hence, in what follows, we refer to the minimization of (5.2) as the inner optimization problem.

We use the standard variational approach to derive optimality conditions for (5.2) with fixed design $\boldsymbol{w}$. The Lagrangian functional $\mathscr{L}^{\mathrm{I}}: \mathscr{V}_{g} \times \mathscr{E} \times \mathscr{V}_{0} \rightarrow \mathbb{R}$ is given by

$$
\mathscr{L}^{\mathrm{I}}(u, m, p):=\mathcal{J}(m, \boldsymbol{w} ; \mathbf{d})+\left\langle e^{m} \nabla u, \nabla p\right\rangle-\langle f, p\rangle-\langle p, h\rangle_{\Gamma_{N}} .
$$


Here, $p \in \mathscr{V}_{0}$ is the Lagrange multiplier and we use the superscript $I$ to emphasize that the Lagrangian corresponds to the inner optimization problem. The formal Lagrange multiplier method [35] yields that, at a minimizer of (5.2), variations of the Lagrangian functional with respect to all variables vanish, which yields

$$
\begin{aligned}
\left\langle e^{m} \nabla u, \nabla \tilde{p}\right\rangle-\langle f, \tilde{p}\rangle-\langle\tilde{p}, h\rangle_{\Gamma_{N}} & =0, \\
\left\langle e^{m} \nabla \tilde{u}, \nabla p\right\rangle+\left\langle\mathcal{B}^{*} \mathbf{W}_{\sigma}(\mathcal{B} u-\mathbf{d}), \tilde{u}\right\rangle & =0, \\
\left\langle m-m_{\mathrm{pr}}, \tilde{m}\right\rangle_{\mathscr{E}}+\left\langle\tilde{m} e^{m} \nabla u, \nabla p\right\rangle & =0,
\end{aligned}
$$

for all variations $(\tilde{u}, \tilde{m}, \tilde{p}) \in \mathscr{V}_{0} \times \mathscr{E} \times \mathscr{V}_{0}$. Note that (5.4a), (5.4b) and (5.4c) are the weak forms of the state, the adjoint and the gradient equations, respectively. The left hand side of (5.4c) is the gradient for the cost functional (5.2), provided that $u$ and $p$ are solutions to the state and adjoint equations, respectively $[6,35]$.

5.2. Hessian-vector application. To evaluate the OED objective function (4.5), systems of the form $\mathcal{H} y=z$ have to be solved, where $\mathcal{H}$ is the Hessian with respect to $m$ of the regularized data misfit functional defined in (5.2). Using second variations of $\mathscr{L}^{\mathrm{I}}$ defined in (5.3) allows derivation of expressions for $\mathcal{H} y=z$. For $z \in \mathscr{H} \subset \mathscr{E}^{\prime}$, the solution $y \in \mathscr{E}$ of $\mathcal{H} y=z$ is obtained by solving a coupled system of PDEs: Find $(v, q, y) \in \mathscr{V}_{0} \times \mathscr{V}_{0} \times \mathscr{E}$ such that for all $(\tilde{p}, \tilde{u}, \tilde{y}) \in \mathscr{V}_{0} \times \mathscr{V}_{0} \times \mathscr{E}$ the following equations are satisfied:

$$
\begin{aligned}
\left\langle e^{m} \nabla v, \nabla \tilde{p}\right\rangle+\left\langle y e^{m} \nabla u, \nabla \tilde{p}\right\rangle & =0, \\
\left\langle\mathcal{B}^{*} \mathbf{W}_{\sigma} \mathcal{B} v, \tilde{u}\right\rangle+\left\langle y e^{m} \nabla \tilde{u}, \nabla p\right\rangle+\left\langle e^{m} \nabla \tilde{u}, \nabla q\right\rangle & =0, \\
\left\langle\tilde{y} e^{m} \nabla v, \nabla p\right\rangle+\langle y, \tilde{y}\rangle_{\mathscr{E}}+\left\langle\tilde{y} y e^{m} \nabla u, \nabla p\right\rangle+\left\langle\tilde{y} e^{m} \nabla u, \nabla q\right\rangle & =\langle z, \tilde{y}\rangle .
\end{aligned}
$$

The equations (5.5a) and (5.5b) are sometimes called incremental state and adjoint equations, respectively, and the left hand side in (5.5c) describes the application of the Hessian to a vector $y$. In practice, $\mathcal{H} y=z$ is solved iteratively using a Krylov method, which requires only the application of $\mathcal{H}$ to vectors. This application can be computed by first solving (5.5a) for $v$, then solving (5.5b) for $q$, and then using these solutions in (5.5c). Next, we provide explicit expressions for the OED problem for the inference of the log coefficient field in (5.1).

5.3. The OED problem as a PDE-constrained optimization problem. Specializing the A-optimal experimental design problem $(\mathcal{P})$ for the problem of inference of the log coefficient field in (5.1) we obtain,

$$
\min _{\boldsymbol{w} \in[0,1]^{n_{s}}} \frac{1}{n_{\mathrm{d}} n_{\mathrm{tr}}} \sum_{i=1}^{n_{\mathrm{d}}} \sum_{k=1}^{n_{\mathrm{tr}}}\left\langle z_{k}, y_{i k}\right\rangle+\gamma P(\boldsymbol{w})
$$

where for $i=1, \ldots, n_{\mathrm{d}}$ and $k=1, \ldots, n_{\mathrm{tr}}$

$$
\begin{aligned}
\left\langle e^{m_{i}} \nabla u_{i}, \nabla \tilde{p}\right\rangle-\langle f, \tilde{p}\rangle-\langle\tilde{p}, h\rangle_{\Gamma_{N}} & =0, & & \forall \tilde{p} \in \mathscr{N}, \\
\left\langle e^{m_{i}} \nabla \tilde{u}, \nabla p_{i}\right\rangle+\left\langle\mathcal{B}^{*} \mathbf{W}_{\sigma}\left(\mathcal{B} u_{i}-\mathbf{d}_{i}\right), \tilde{u}\right\rangle & =0, & & \forall \tilde{u} \in \mathscr{V}, \\
\left\langle m_{i}-m_{\mathrm{pr}}, \tilde{m}\right\rangle_{\mathscr{E}}+\left\langle\tilde{m} e^{m_{i}} \nabla u_{i}, \nabla p_{i}\right\rangle & =0, & & \forall \tilde{m} \in \mathscr{E}, \\
\left\langle\mathcal{B}^{*} \mathbf{W}_{\sigma} \mathcal{B} v_{i k}, \tilde{u}\right\rangle+\left\langle y_{i k} e^{m_{i}} \nabla \tilde{u}, \nabla p_{i}\right\rangle+\left\langle e^{m_{i}} \nabla \tilde{u}, \nabla q_{i k}\right\rangle & =0, & & \forall \tilde{u} \in \mathscr{V}, \\
\left\langle\tilde{y} e^{m_{i}} \nabla v_{i k}, \nabla p_{i}\right\rangle+\left\langle y_{i k}, \tilde{y}\right\rangle_{\mathscr{E}}+\left\langle\tilde{y} y_{i k} e^{m_{i}} \nabla u_{i}, \nabla p_{i}\right\rangle & & & \\
+\left\langle\tilde{y} e^{m_{i}} \nabla u_{i}, \nabla q_{i k}\right\rangle & =\left\langle z_{k}, \tilde{y}\right\rangle, & & \forall \tilde{y} \in \mathscr{E},
\end{aligned}
$$




$$
\left\langle e^{m_{i}} \nabla v_{i k}, \nabla \tilde{p}\right\rangle+\left\langle y_{i k} e^{m_{i}} \nabla u_{i}, \nabla \tilde{p}\right\rangle=0, \quad \forall \tilde{p} \in \mathscr{N}_{0} .
$$

The PDE constraints (5.6b)-(5.6d) are the optimality system (5.4a) $-(5.4 \mathrm{c})$ characterizing the MAP point $m_{i}=m_{\mathrm{MAP}}\left(\boldsymbol{w} ; \mathbf{d}_{i}\right)$. The equations $(5.6 \mathrm{e})-(5.6 \mathrm{~g})$ are the PDE constraints that describe $\mathcal{H}\left(m_{\mathrm{MAP}}\left(\boldsymbol{w} ; \mathbf{d}_{i}\right), \boldsymbol{w} ; \mathbf{d}_{i}\right) y_{i k}=z_{k}$ for $z_{k} \in \mathscr{H}$. Note also that compared to (5.5), we have re-ordered the equations (5.6e)-(5.6g). While (5.5) follows the order in which the Hessian application is computed in practice, the order in (5.6e)$(5.6 \mathrm{~g})$ is such that the linear (block-)operator on the left hand side is symmetric. In summary, (5.6) is a PDE-constrained optimization problem, where the constraints are the first-order optimality conditions of a PDE-constrained inverse problem, and a set of PDEs describing the application of the inverse Hessian to vectors.

5.4. Evaluation and gradient computation of the OED objective. Evaluating the OED objective function in (5.6) involves the following steps: (1) find $\left(u_{i}, m_{i}, p_{i}\right)$ that satisfy equations $(5.6 \mathrm{~b})-(5.6 \mathrm{~d})$ and $(2)$ find $\left(v_{i k}, y_{i k}, q_{i k}\right)$ that satisfy $(5.6 \mathrm{e})-(5.6 \mathrm{~g})$, for $i \in\left\{1, \ldots, n_{\mathrm{d}}\right\}$ and $k \in\left\{1, \ldots, n_{\mathrm{tr}}\right\}$.

To solve the optimization problem (5.6), we rely on gradient-based optimization methods. Thus we need efficient methods for computing the gradient of $\hat{\Psi}$ with respect to the design vector $\boldsymbol{w}$. Again we follow a Lagrangian approach, and employ adjoint variables (i.e., Lagrange multipliers) to enforce the PDE constraints (5.6b)-(5.6g) in the OED problem. The derivation of expressions for the gradient is rather involved, and it deferred to Appendix B. Below, we simply present the final expression for the gradient, which takes the form:

$$
\hat{\Psi}^{\prime}(\boldsymbol{w})=\sum_{i=1}^{n_{\mathrm{d}}} \boldsymbol{\Gamma}_{\text {noise }}^{-1}\left(\mathcal{B} u_{i}-\mathbf{d}_{i}\right) \odot \mathcal{B} p_{i}^{*}-\frac{1}{n_{\mathrm{d}} n_{\text {tr }}} \sum_{i=1}^{n_{\mathrm{d}}} \sum_{k=1}^{n_{\text {tr }}} \boldsymbol{\Gamma}_{\text {noise }}^{-1} \mathcal{B} v_{i k} \odot \mathcal{B} v_{i k}
$$

where $u_{i}, v_{i k}$ are available from the evaluation of $\hat{\Psi}$ as described above, $\odot$ denotes the Hadamard product, ${ }^{1}$ and, for $i \in\left\{1, \ldots, n_{\mathrm{d}}\right\}$, the $p_{i}^{*}$ are obtained by solving the following systems for the OED adjoint variables $\left(p_{i}^{*}, m_{i}^{*}, u_{i}^{*}\right) \in \mathscr{V}_{0} \times \mathscr{E} \times \mathscr{V}_{0}$ :

$$
\begin{aligned}
\left\langle\mathcal{B}^{*} \mathbf{W}_{\sigma} \mathcal{B} p_{i}^{*}, \tilde{u}\right\rangle+\left\langle m_{i}^{*} e^{m_{i}} \nabla \tilde{u}, \nabla p_{i}\right\rangle+\left\langle e^{m_{i}} \nabla \tilde{u}, \nabla u_{i}^{*}\right\rangle & =\left\langle b_{i}^{1}, \tilde{u}\right\rangle, \\
\left\langle\tilde{m} e^{m_{i}} \nabla p_{i}, \nabla p_{i}^{*}\right\rangle+\left\langle m_{i}^{*}, \tilde{m}\right\rangle_{\mathscr{E}}+\left\langle\tilde{m} m_{i}^{*} e^{m_{i}} \nabla u_{i}, \nabla p_{i}\right\rangle+\left\langle\tilde{m} e^{m_{i}} \nabla u_{i}, \nabla u_{i}^{*}\right\rangle & =\left\langle b_{i}^{2}, \tilde{m}\right\rangle, \\
\left\langle e^{m_{i}} \nabla \tilde{p}, \nabla p_{i}^{*}\right\rangle+\left\langle m_{i}^{*} e^{m_{i}} \nabla u_{i}, \nabla \tilde{p}\right\rangle & =\left\langle b_{i}^{3}, \tilde{p}\right\rangle,
\end{aligned}
$$

for all $(\tilde{u}, \tilde{m}, \tilde{p}) \in \mathscr{V}_{0} \times \mathscr{E} \times \mathscr{V}_{0}$, with the right hand sides given by

$$
\begin{aligned}
& \left\langle b_{i}^{1}, \tilde{u}\right\rangle=\frac{1}{n_{\mathrm{d}} n_{\mathrm{tr}}} \sum_{k=1}^{n_{\mathrm{tr}}}\left[2\left\langle y_{i k} e^{m_{i}} \nabla \tilde{u}, \nabla q_{i k}\right\rangle+\left(y_{i k}^{2} e^{m_{i}} \nabla \tilde{u}, \nabla p_{i}\right)\right], \\
& \left\langle b_{i}^{2}, \tilde{m}\right\rangle=\frac{1}{n_{\mathrm{d}} n_{\mathrm{tr}}} \sum_{k=1}^{n_{\mathrm{tr}}}\left[2\left\langle\tilde{m}_{i} e^{m_{i}} \nabla v, \nabla q\right\rangle+2\left\langle\tilde{m} y_{i k} e^{m_{i}} \nabla u_{i}, \nabla q_{i k}\right\rangle\right. \\
& \left.+2\left\langle\tilde{m} y_{i k} e^{m_{i}} \nabla v_{i k}, \nabla p_{i}\right\rangle+\left\langle\tilde{m} y_{i k}^{2} e^{m_{i}} \nabla u_{i}, \nabla p_{i}\right\rangle\right], \\
& \left\langle b_{i}^{3}, \tilde{p}\right\rangle=\frac{1}{n_{\text {tr }} n_{\mathrm{d}}} \sum_{k=1}^{n_{\text {tr }}}\left[2\left\langle y_{i k} e^{m_{i}} \nabla \tilde{p}, \nabla v_{i k}\right\rangle+\left\langle y_{i k}^{2} e^{m_{i}} \nabla u_{i}, \nabla \tilde{p}\right\rangle\right] .
\end{aligned}
$$

Note that the linear operator on the left hand side of (5.8) coincides with the left hand side operator in (5.6e)-(5.6g), after proper identification of variables. The fact

\footnotetext{
${ }^{1}$ For vectors $\boldsymbol{x}$ and $\boldsymbol{y}$ in $\mathbb{R}^{n}$, the Hadamard product, $\boldsymbol{x} \odot \boldsymbol{y}$, is a vector in $\mathbb{R}^{n}$ with components $(\boldsymbol{x} \odot \boldsymbol{y})_{i}=x_{i} y_{i}, i=1, \ldots, n$.
} 
that the system for the OED adjoint variables coincides with the system describing the Hessian of the inner optimization problem, i.e., the Hessian of $\mathcal{J}$, defined in (5.2), with respect to $m$ can be exploited in numerical computations. In particular, if a Newton solver for the inner optimization problem is available, the implementation can easily be adapted to perform the computations required to evaluate the OED objective, and to compute the OED gradient. We summarize the steps for computing the OED objective function and its gradient in Algorithm 1.

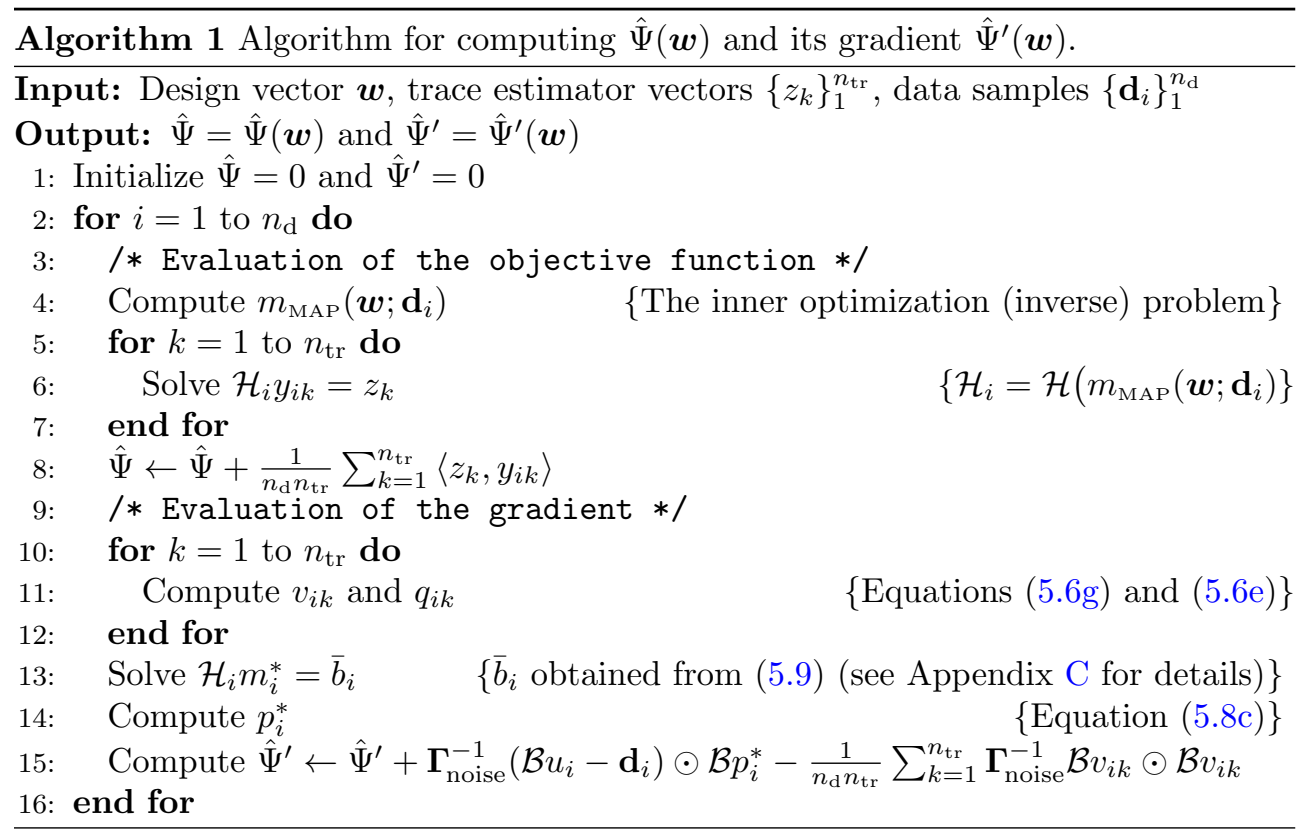

5.5. Scalability of the OED solver. Here, we provide a discussion of the computational complexity and resulting scalability of solving the OED problem (5.6). Although this discussion is qualitative in nature, we do provide numerical evidence of the scalability of our OED solver in section 6 . The cost of solving the OED problem in measured in terms of the number of required forward-like PDE solves, i.e., solves of (5.1), or its adjoint or incremental variants. We measure cost in this way to remain agnostic to the specific governing forward PDEs and the particular PDE solver employed. These forward-like PDE solves constitute the kernel component of the OED optimization solver, and for any non-trivial PDE forward problem, the PDE solves overwhelmingly dominate the overall cost; the remaining linear algebra is negligible in comparison. Having defined cost in this manner, scalability then requires that the number of forward-like PDE solves is independent of problem dimensions, which for the OED problem (5.6) are the (discretized) parameter dimension and the sensor dimension $n_{s}$ (the state dimension is hidden within the forward-like PDE solver).

To assert scalability of the OED solver, we have to argue that (1) the evaluation of the OED objective $\hat{\Psi},(2)$ the evaluation of the gradient of the OED objective $\hat{\Psi}^{\prime}$, and (3) the number of OED optimization iterations are all independent of the parameter and sensor dimensions. To make this argument, we begin by identifying a property of the Hessian systems that are solved at each OED optimization iteration. These Hessian systems include those arising at each iteration of the inner optimization 
problem (i.e., minimizing $\mathcal{J}$ in (5.2)), as well as the Hessian solves characterizing the posterior covariance in the OED objective evaluation $((5.6 \mathrm{e})-(5.6 \mathrm{~g}))$ and those arising in OED gradient computation (5.8). Consider the Hessian $\mathcal{H}$ evaluated at the MAP point and notice that $\mathcal{H}$ can be written as $\mathcal{H}=\mathcal{H}_{\text {misfit }}+\mathcal{C}_{\mathrm{pr}}^{-1}$, with $\mathcal{H}_{\text {misfit }}$ representing the Hessian of the first term (i.e., the data misfit term) in $\mathcal{J}$ defined in (2.5). As discussed in $[7,15]$, the numerical rank $r$ of the prior-preconditioned data misfit Hessian, $\tilde{\mathcal{H}}_{\text {misfit }}=\mathcal{C}_{\mathrm{pr}}^{1 / 2} \mathcal{H}_{\text {misfit }} \mathcal{C}_{\mathrm{pr}}^{1 / 2}$, is independent of the parameter dimension and, for many inverse problems, small. Moreover, the rank is independent of the sensor dimension as well. This parameter/sensor dimension-independence of $r$ reflects the fact that (1) the data are often finite-dimensional, (2) the parameter-to-observable map is often smoothing, and (3) the prior covariance operator is of smoothing type. The numerical rank $r$ depends on the parameter-to-observable map, the smoothing properties of the prior, and the true information content of the data. The rank grows initially with parameter and sensor dimensions until all information contained in the data about the parameters has been resolved. Beyond this, the rank $r$ of $\tilde{\mathcal{H}}_{\text {misfit }}$ is insensitive to further increases in parameter and sensor dimension (e.g., through mesh refinement).

Next, we analyze the computational cost (again, measured in forward-like PDE solves) of evaluation of the OED objective function and its gradient as detailed in Algorithm 1. We rely on inexact Newton-CG with Armijo line search to solve the inner optimization problems in step 4 of the algorithm. The computational cost of each Newton step is dominated by the conjugate gradient iterations. Using the prior covariance as a preconditioner for $\mathrm{CG}$, the number of $\mathrm{CG}$ iterations will be $\mathcal{O}(r)$ (see [8] for mesh invariance properties of CG for operators that are compact perturbations of the identity). Each CG iteration involves an application of the data misfit Hessian, which in turn involves a pair of incremental forward/adjoint PDE solves; therefore, the cost, in terms of forward-like PDE solves, of each inner optimization problem is $\mathcal{O}\left(n_{\text {newton }} \times 2 \times r\right)$, where $n_{\text {newton }}$ is the total number of Newton iterations. Note that here we do not take into account the inexactness of Newton-CG. If in earlier iterations the Newton system is solved only approximately, $n_{\text {newton }}$ can be replaced with a smaller number. Next, we note that for each data sample $\mathbf{d}_{i}, i=1 \ldots n_{\mathrm{d}}$, we perform $n_{\mathrm{tr}}$ Hessian solves in steps 5-7 of the algorithm, where we solve for $y_{i k}$, $k=1, \ldots, n_{\mathrm{tr}}$. Thus, since we use CG to solve these systems, it follows that the computational cost, measured in forward-like PDE solves, of evaluating the OED objective function is

$$
\mathcal{O}\left(n_{\mathrm{d}} \times n_{\text {newton }} \times 2 \times r\right)+\mathcal{O}\left(n_{\mathrm{d}} \times n_{\text {tr }} \times 2 \times r\right) .
$$

Note also that by the mesh invariance properties of the Newton method for nonlinear optimization [14], $n_{\text {newton }}$ is independent of the parameter dimension.

To compute the gradient, we need to perform the computations in step 11, which entail $2 \times n_{\mathrm{d}} \times n_{\text {tr }}$ PDE solves, as well as the Hessian solves in step 13 of the Algorithm 1, whose cost is $\mathcal{O}\left(n_{\mathrm{d}} \times 2 \times r\right)$ PDE solves. Thus, the cost of evaluating the OED gradient is

$$
2 \times n_{\mathrm{tr}} \times n_{\mathrm{d}}+\mathcal{O}\left(n_{\mathrm{d}} \times 2 \times r\right)
$$

forward-like PDE solves.

Observe that step 6 of Algorithm 1 involves $n_{\text {tr }}$ systems with the same Hessian operator and different right hand sides. Thus, it is possible to further reduce the complexity of the algorithm. For instance, precomputing a low rank approximation of the 
prior-preconditioned data misfit Hessian $\tilde{\mathcal{H}}_{\text {misfit }}$ (after solving the inner optimization problem) provides an efficient method for applications of the inverse Hessian that is free of PDE solves $[7,15]$. Using this low rank approximation of $\tilde{\mathcal{H}}_{\text {misfit }}$ allows us to remove the factor $n_{\mathrm{tr}}$ in the second term of (5.10).

The final argument to make is that the number of OED optimization iterations is parameter/sensor dimension-independent. If one solves the OED problem (5.6) using a Newton method, we would expect this to be the case. In the example of section 6 , we employ a quasi-Newton method. It is difficult to make a dimension-independence argument for quasi-Newton for the OED problem; however, in that section we do observe dimension independence of OED optimization iterations.

5.6. Sparsity control. Here we briefly comment on the sparsity enforcing penalty method used in the present work, which is based on the approach in [1]. In particular, considering the problem $(\mathcal{P})$, we first solve the problem with $P(\boldsymbol{w})=\mathbf{1}^{T} \boldsymbol{w}$, amounting to an $\ell^{1}$ penalty to obtain the minimizer $\boldsymbol{w}_{0}^{*}$. Subsequently, we consider a sequence of penalty functions, $P_{\varepsilon}(\boldsymbol{w})$ such that as $\varepsilon \rightarrow 0, P_{\varepsilon}$ approaches the $\ell^{0}$ norm. To cope with the non-convexity of these penalty functions, we follow a continuation strategy, i.e., we decrease $\left\{\varepsilon_{i}\right\}$ : For $\varepsilon_{1}$, we solve $(\mathcal{P})$ with penalty function $P_{\varepsilon_{1}}$ and the initial guess (for the optimization algorithm) given by the $\boldsymbol{w}_{0}^{*}$. Subsequently, for each $i \geq 2$ the problem is solved with $P_{\varepsilon_{i}}$ as the penalty function and the initial guess given by the solution of the proceeding optimization problem corresponding to $\varepsilon_{i-1}$. The precise definition of the penalty functions $P_{\varepsilon}$ used follows [1]. In practice, we observe that a few continuation iterations are sufficient to attain an optimal weight vector with a $0 / 1$ structure.

6. Example 1: Idealized subsurface flow. In this section, we study the effectiveness of our OED approach applied to the parameter estimation problem considered in section 5. We interpret (5.1) as subsurface flow problem and thus refer to $u$ as pressure and to $m$ as $\log$ permeability.

6.1. Setup of forward problem. To detail the forward problem (5.1), we consider the domain $\mathcal{D}:=(0,1) \times(0,1) \subset \mathbb{R}^{2}$ and no volume forcing, i.e., $f=0$. We assume no-outflow conditions on $\Gamma_{N}:=\{0,1\} \times(0,1)$, i.e., the homogeneous Neumann conditions $e^{m} \nabla u \cdot \boldsymbol{n}=0$ on $\Gamma_{N}$. The flow is driven by a pressure difference between the top and the bottom boundary, i.e., we use $u=1$ on $(0,1) \times\{1\}$ and $u=0$ on $(0,1) \times\{0\}$. This Dirichlet part of the boundary is denoted by $\Gamma_{D}:=(0,1) \times\{0,1\}$. In Figure 6.1, we show the "truth" permeability used in our numerical tests, the corresponding pressure and the Darcy velocity field.
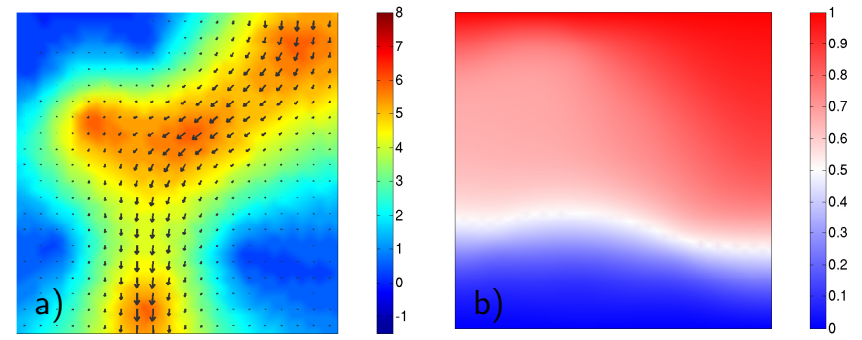

FIG. 6.1. The color in (a) shows the log permeability field $m_{\text {true }}$, and the arrows depict the Darcy velocity field, $\boldsymbol{q}=-\frac{e^{m}}{\eta} \nabla u$, where $m=m_{\text {true }}$ and $\eta$ is the viscosity, here assumed to be $\eta=1$. The pressure field $u$ obtained by solving the state equation with $m_{\text {true }}$ is shown in (b). 

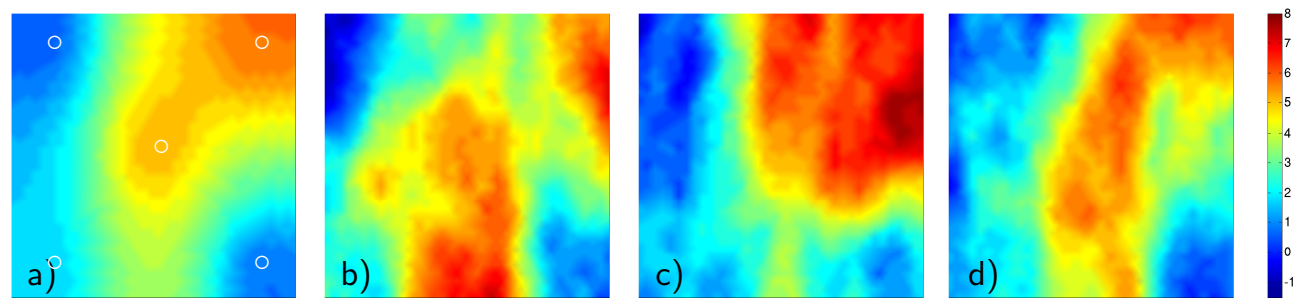

FIG. 6.2. Prior mean log permeability $m_{p r}$ with circles indicating the points $\boldsymbol{x}_{1}, \ldots, \boldsymbol{x}_{5}$ where permeability measurements are available (a), and samples drawn from the prior distribution (b)-(d).

6.2. Prior and noise model. We assume given estimates $m_{\text {true }}^{1}, \ldots, m_{\text {true }}^{5}$ of the $\log$ permeability at five points, i.e., $N=5$, in $\mathcal{D}$, namely $\boldsymbol{x}_{1}=(0.1,0.1)$, $\boldsymbol{x}_{2}=(0.1,0.9), \boldsymbol{x}_{3}=(0.9,0.1), \boldsymbol{x}_{4}=(0.9,0.9)$, and $\boldsymbol{x}_{5}=(0.5,0.5)$. Based on this knowledge, we compute $m_{\mathrm{pr}}$, the mean of the prior measure, as a regularized least-squares fit of these point observations by solving

$$
m_{\mathrm{pr}}=\underset{m \in \mathscr{E}}{\arg \min } \frac{1}{2}\langle m, \mathcal{A} m\rangle+\frac{\alpha}{2} \sum_{i=1}^{N} \int_{\mathcal{D}} \delta_{i}(\boldsymbol{x})\left[m(\boldsymbol{x})-m_{\text {true }}(\boldsymbol{x})\right]^{2} d \boldsymbol{x} .
$$

Here, $\mathcal{A}[m]=-\nabla \cdot(\boldsymbol{\Theta} \nabla m)$, where the positive definite matrix $\boldsymbol{\Theta}$ allows to control the prior covariance. We define the prior covariance as $\mathcal{C}_{\mathrm{pr}}:=\mathcal{L}^{-2}$, where $\mathcal{L}=$ $\mathcal{A}+\alpha \sum_{i=1}^{N} \delta_{i}$, where we use the following parameter values:

$$
\alpha=1, \quad \Theta=5 \times 10^{-2}\left(\begin{array}{cc}
1 / 2 & 0 \\
0 & 2
\end{array}\right) .
$$

In Figure 6.2, we show the prior mean $m_{\mathrm{pr}}$, obtained by solving (6.1) and three random draws from the prior distribution. Note that our choice for $\Theta$ corresponds to a prior distribution with stronger correlation in $y$-direction. It remains to specify the noise covariance matrix, for which we choose $\boldsymbol{\Gamma}_{\text {noise }}=\sigma^{2} I$, with $\sigma=0.05$. We use a linear triangular finite element mesh with $n=1,121$ degrees of freedom to discretize the state, adjoint and the parameter variables. The discrete inference parameters are the coefficients in the finite element expansion of the parameter field.

6.3. Effectiveness of A-optimal design. We solve the OED problem (5.6a) with $n_{\mathrm{d}}=5$ experimental data samples $\mathbf{d}_{i}$, and use $n_{\mathrm{tr}}=20$ random vectors in the trace estimator. We employ $\ell_{0}$-sparsification using the continuation process described in section 5.6. We obtain an optimal sensor configuration with 10 sensors for the penalty parameter $\gamma=0.008$, and an optimal design with 20 sensors for $\gamma=0.005$. As first test of the effectiveness of the resulting designs, we solve the inference problem with the "truth" parameter field given in Figure 6.1a). Using data obtained at the A-optimal sensor configuration (with 10 sensors), we compute the MAP point by solving (5.2) and the Gaussian approximation of the posterior measure at the MAP point. The results are shown in Figure 6.3, where the posterior standard deviation field is also compared with the prior standard deviation field. To study the effectiveness of the optimal designs, we first report the error with respect to the "truth" permeability field $m_{\text {true }}$. In Figure 6.4 we show a comparison of the relative error of the MAP 

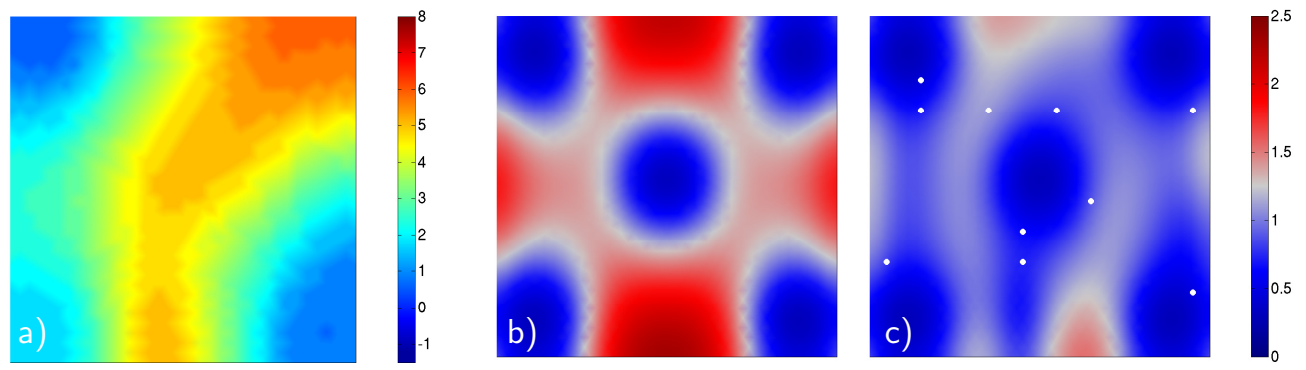

FIG. 6.3. MAP point computed using the optimal design (a); prior standard deviation field (b) and posterior standard deviation field, with the optimal design sensor locations (10 sensors) indicated by white dots (c).

estimator,

$$
E_{\text {rel }}(\boldsymbol{w})=\frac{\left\|m_{\mathrm{MAP}}(\boldsymbol{w})-m_{\text {true }}\right\|}{\left\|m_{\text {true }}\right\|},
$$

and $\operatorname{of} \operatorname{tr}\left(\mathcal{H}(\boldsymbol{w})^{-1}\right)=\operatorname{tr}\left(\boldsymbol{\Gamma}_{\text {post }}\right)$ for the optimal design $\boldsymbol{w}_{\text {opt }}$ and for random designs with the same number of sensor locations, where $\|\cdot\|$ is the $L^{2}$-norm. From Figure 6.3, we draw the following conclusions: (1) The optimal design with 10 sensors improves over randomly selected designs more significantly than the optimal design with 20 sensors; this indicates that as sensors become more scarce, computing optimal design is more important. (2) There is a correlation between minimizing the average variance and that of minimizing the $L^{2}$-error of the MAP estimator. This is interesting but not entirely surprising, because for a Bayesian linear inverse problem with Gaussian prior and noise, it can be shown that minimizing the average posterior variance is equivalent to minimizing the average mean square error of the MAP estimator [1]. Note that the results shown in Figure 6.4 study the effectiveness of the OED with
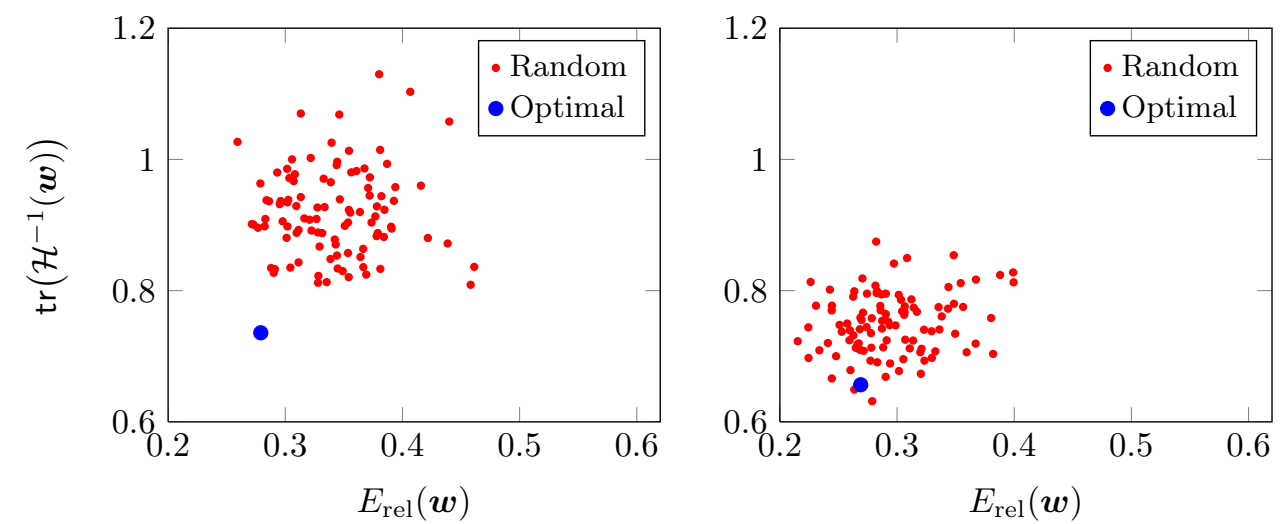

FIG. 6.4. Shown is the relative error, $E_{\text {rel }}$, of the MAP estimator versus $\operatorname{tr}\left(\mathcal{H}^{-1}\left(m_{\text {MAP }}(\boldsymbol{w})\right)\right)$ for random designs $\boldsymbol{w}$ (red dots) and the optimal design $\boldsymbol{w}_{\text {opt }}$ (blue dot). These results are for designs with 10 (left) and 20 (right) sensors.

respect to a specific "truth" model. A natural question to ask is how effective the design is if we were trying to recover a different underlying truth? To address this 
issue, we conduct a statistical test of the effectiveness of the optimal designs as follows. We draw samples $\left\{m_{1}^{\prime}, \ldots, m_{n_{d}^{\prime}}^{\prime}\right\}$ from the prior measure and get corresponding data vectors $\mathbf{d}_{i}^{\prime}=f\left(m_{i}^{\prime}\right)+\boldsymbol{\eta}_{i}^{\prime}$, with $\eta_{i}^{\prime}$ drawn from $\mathcal{N}\left(\mathbf{0}, \boldsymbol{\Gamma}_{\text {noise }}\right), i=1, \ldots, n_{d}^{\prime}$. For a given design, $\boldsymbol{w}$, we compute an expected error $\bar{E}_{\text {rel }}$ and an expected average variance $\bar{V}$ :

$$
\bar{V}(\boldsymbol{w})=\frac{1}{n_{d}^{\prime}} \sum_{i=1}^{n_{d}^{\prime}} \operatorname{tr}\left(\mathcal{H}^{-1}\left(\boldsymbol{w}, \mathbf{d}_{i}^{\prime}\right)\right), \quad \bar{E}_{\mathrm{rel}}(\boldsymbol{w})=\frac{1}{n_{d}^{\prime}} \sum_{i=1}^{n_{d}^{\prime}} \frac{\left\|m_{\mathrm{MAP}}\left(\boldsymbol{w} ; \mathbf{d}_{i}^{\prime}\right)-m_{i}^{\prime}\right\|}{\left\|m_{i}^{\prime}\right\|} .
$$

For the purpose of this numerical test, we let $n_{d}^{\prime}$ be larger than the number $n_{\mathrm{d}}$ of the data samples used in computing the optimal design, and the samples $\left\{m_{1}^{\prime}, \ldots, m_{n_{d}^{\prime}}^{\prime}\right\}$ are drawn independently of the samples used in the sample average used for the OED objective function (see section 4.3). Hence, $\bar{V}$ is essentially a more accurate estimate of the objective function we sought to minimize when solving the OED problem. This allows us to assess how well an optimal design, computed based on a small set of data $\left\{\mathbf{d}_{1}, \ldots, \mathbf{d}_{n_{\mathrm{d}}}\right\}$ does in minimizing the more accurate estimate $\bar{V}$.

For designs with 10 and 20 sensors, we compute $\bar{V}(\boldsymbol{w})$ and $\bar{E}_{\text {rel }}(\boldsymbol{w})$ with $n_{d}^{\prime}=50$ for optimal and for $n_{w}=30$ randomly chosen designs $\boldsymbol{w}_{1}, \ldots, \boldsymbol{w}_{n_{w}}$. The results, shown in Figure 6.5, indicate that the A-optimal designs computed with a relatively small number of data samples not only minimize the average posterior variance, but also result in a minimal expected error between the true parameter and the MAP point.
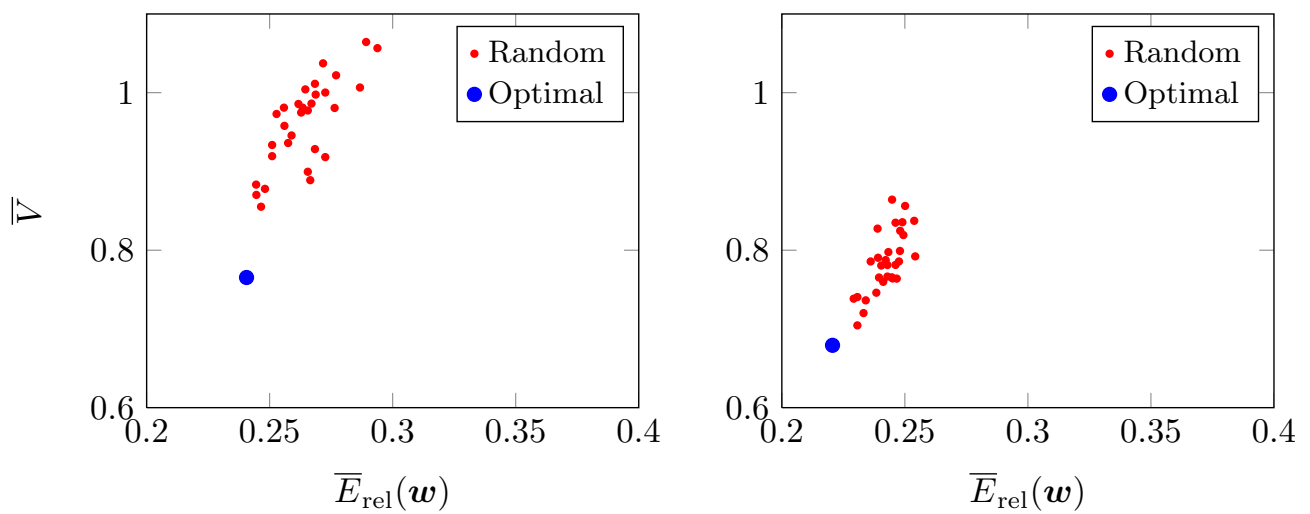

FIG. 6.5. Expected relative error $\bar{E}_{\text {rel }}(\boldsymbol{w})$ versus expected average variance $\bar{V}(\boldsymbol{w})$ for random designs (red dots) and for the optimal design (blue dot). The left and right panels correspond to designs with 10 and 20 sensors, respectively.

6.4. Scalability and performance. Finally, we examine the convergence behavior of our method as the number of parameters and the number of sensor candidate locations increases. Specifically, we study the computational cost in terms of the number of solves of (5.1), its adjoint, or the associated incrementals. These elliptic PDE solves are the main building block of our method.

First, we consider the cost of computing the OED objective function and its gradient. As seen in Algorithm 1 and the discussion in section 5.5, a significant part of the computational cost of evaluating the OED objective function amounts to solving the inner optimization problem for the MAP point using an inexact NewtonCG method. Here, the computational cost is dominated by the CG iterations needed 
in each Newton step. Hence, as a measure of the computational cost, we report the total number of "inner" CG iterations. We also report the number of "outer" CG iterations in steps 6 and 13 of Algorithm 1, which are required for computing the OED objective function and the gradient, respectively. For this numerical study, we focused on the evaluation of the OED cost function and its gradient at $\boldsymbol{w}=(1,1, \cdots, 1) \in \mathbb{R}^{n_{s}}$ (i.e. with all sensors active) and with $n_{\mathrm{tr}}=n_{\mathrm{d}}=1$. The results shown in Figure 6.6 indicate that the computational cost of evaluating the OED objective function and its gradient are insensitive to increasing the parameter dimension, and only depend weakly on the number of sensor candidate locations. Figure 6.6 also shows the number of interior point quasi-Newton iterations required for solving the OED optimization problem, as parameter and sensor dimensions increase. As can be seen, the number of iterations for solving the OED optimization problem is insensitive to both parameter and sensor dimensions.

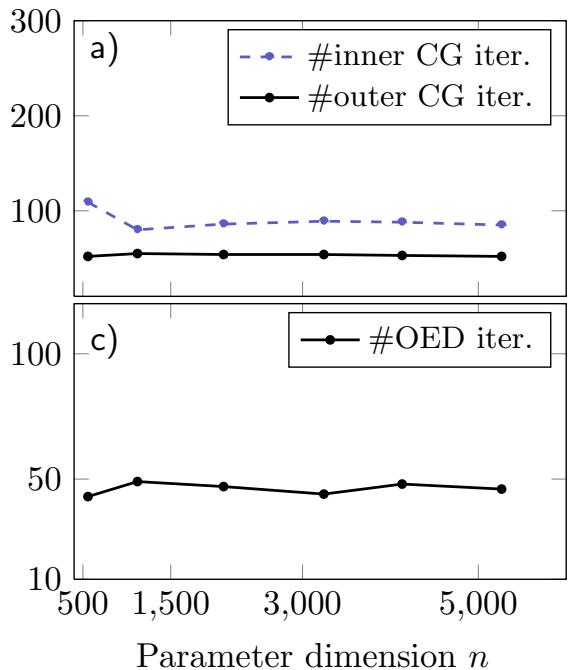

Parameter dimension $n$

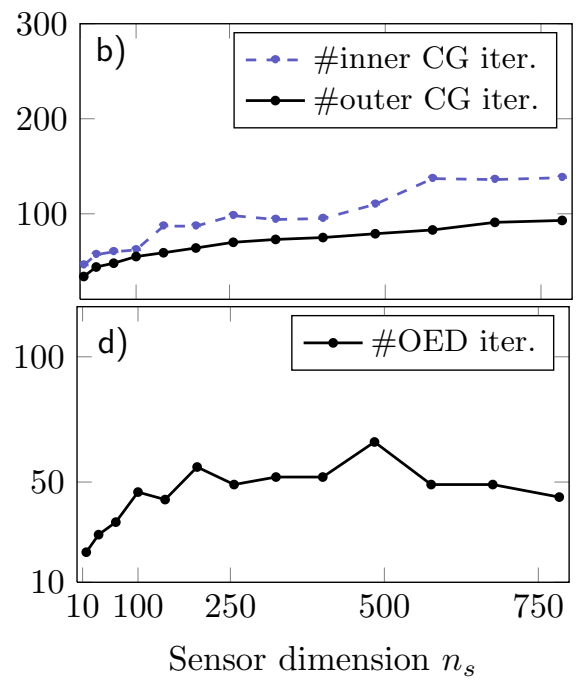

FIG. 6.6. Computational cost of evaluating the OED objective function and its gradient: Shown in (a) and (b) are the total number of CG iterations for the inner optimization problem (to compute the MAP point) and for the outer optimization problem (trace estimation with inverse Hessian) as the parameter dimension increases (a), and as the number of candidate locations for sensors increases (b). Shown in (c) and (d) are the number of interior-point iterations for increasing parameter dimension (c) and sensor dimension (d). For (a) and (c), the sensor dimension is fixed at $n_{s}=100$, and for (b) and (d), the parameter dimension is fixed at $n=2101$.

7. Example 2: Subsurface flow based on SPE10 model. In this section, we consider a more realistic permeability field using permeability field data from the Society of Petroleum Engineers' 10th SPE Comparative Solution Project (SPE10). ${ }^{2}$

7.1. Bayesian inverse problem setup. We define the physical domain $\mathcal{D}=$ $(0,2.2) \times(0,1.2)$ (with unit of length in 1000's of feet) and use as the "truth" permeability field a vertical slice ${ }^{3}$ of the three-dimensional SPE10 permeability data. Following the setup of the SPE10 model, we consider an injection well in the center of the domain, and four production wells at the corners of the domain. The injection

\footnotetext{
${ }^{2}$ See http://www.spe.org/web/csp/datasets/set02.htm for the description of the dataset.

${ }^{3}$ We use the 70th slice, counted from the top.
} 
well is modeled as a mollified point source, and enters (5.1) through the right hand side function $f$ given by $f(\boldsymbol{x})=C /(2 \pi L) \exp \left\{-1 /(2 L)\left(\boldsymbol{x}-\boldsymbol{x}_{0}\right)^{T}\left(\boldsymbol{x}-\boldsymbol{x}_{0}\right)\right\}$, with $L=10^{-4}$ and $C=50$, and $\boldsymbol{x}_{0}=(1.1,0.6)$. To model the production wells, we fix the pressure at zero at the four corners of the domain. Specifically, we cut circular regions from the four corners of the domain (modeling the boundaries of wells) and impose zero Dirichlet boundary conditions on the resulting quarter circles. Homogeneous Neumann boundary conditions are used on the remainder of the boundary. In Figure 7.1, we show the "truth" log permeability field, as well as the Darcy velocity field and the pressure obtained by solving the state equation with the true permeability field.
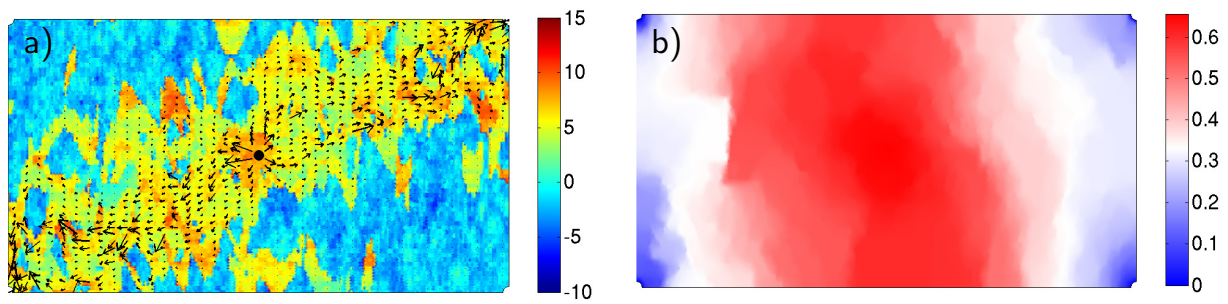

FIG. 7.1. In (a) we show the "truth" SPE10 log permeability field with arrows depicting the corresponding Darcy velocity field, $\boldsymbol{q}=-\frac{e^{m}}{\eta} \nabla u$, where $m=m_{\text {true }}$ denotes the true permeability and $\eta$ is the viscosity, chosen as $\eta=1$. The black dot in the center indicates the location of the injection well. Shown in (b) is the pressure $u$ obtained by solving the state equation with $m_{\text {true }}$.

The prior construction is similar as in the previous test problem. We assume estimates $m_{\text {true }}^{1}, \ldots, m_{\text {true }}^{5}$ of the $\log$ permeability at $N=5$ points, one at the injection well in the center of $\mathcal{D}$, and the others are near each of the four corners of the domain (at the production well boundaries). Based on this data, we compute the mean of the prior measure, as a regularized least-squares fit of these point observations as in (6.1); see Figure 7.2(a). As before, the prior covariance is $\mathcal{C}_{0}=\mathcal{L}^{-2}$ where $\mathcal{L}=-\theta \Delta+\alpha \sum_{i=1}^{N} \delta_{i}$, with parameter values in (6.2) given by $\theta=3.54 \times 10^{-2}$ and $\alpha=1.25 \times 10^{1}$. Linear triangular finite elements with $n=10,202$ degrees of freedom are used to discretize the state, adjoint and the parameter variables.

7.2. A-optimal design of experiments. We use a grid of 128 candidate sensor locations in the domain $\mathcal{D}$, and compute an A-optimal design based on one data sample, computed using one random draw from prior depicted in Figure 7.2(b). For the OED objective function, given in (5.6a), we use a trace estimator with $n_{\mathrm{tr}}=20$ random vectors. After six continuation iterations, our method converged to a $0 / 1$ design vector. In each continuation step we terminated the interior-point iterations if either the relative residual fell below $10^{-5}$ or if we reached a maximum of 100 interior-point BFGS iterations.

We solve the Bayesian inverse problem using experimental data at the A-optimal sensor locations for the "truth" log permeability $m_{\text {true }}$. To capture the extreme variations in the permeability field, we solve the forward problem using quadratic triangular elements on a finer mesh with $n=237,573$ degrees of freedom, and record pressure measurements at the sensor sites. This data vector is subsequently used in the solution of the Bayesian inverse problem. After solving the Bayesian inverse problem with the A-optimal sensor configuration, in Figure 7.2c, we show the MAP point, and in Figure 7.3, compare the prior and posterior standard deviation fields. Finally, to 

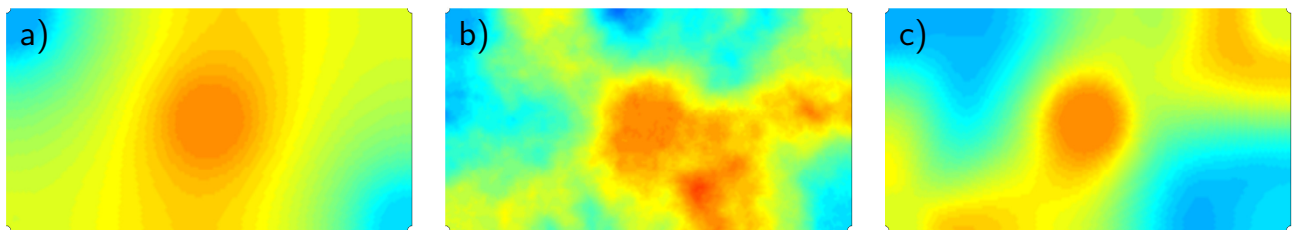

FIG. 7.2. Prior mean (a), sample draw from the prior used to generate data for the OED process (b), and, in (c) the MAP point found with on the optimal sensor locations shown in Figure 7.3b.
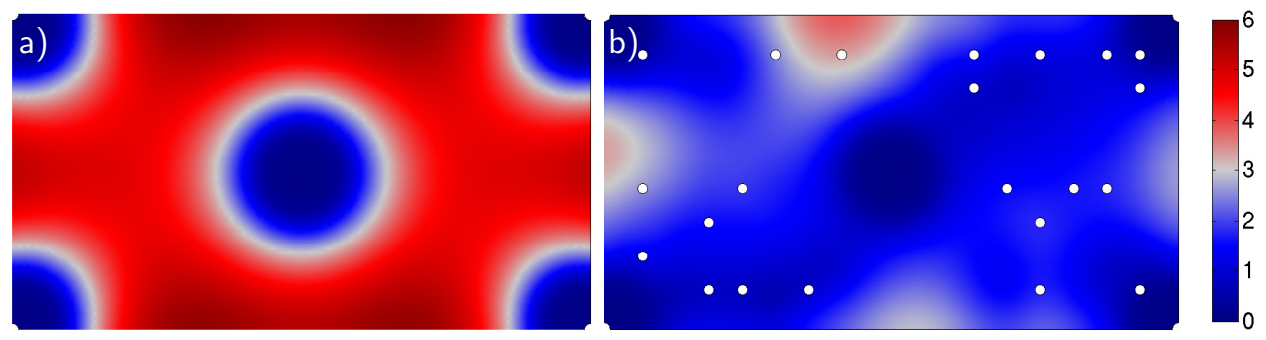

FIG. 7.3. Shown are the prior standard deviation field (a) and the posterior standard deviation field based on solving the Bayesian inverse problem using the A-optimal sensor placement (b). The white dots in (b) indicate the A-optimal sensor locations.

assess the effectiveness of the A-optimal sensor placement computed, we compare the relative error of the MAP point as well as the average posterior variance, based on solving the Bayesian inverse problem using the optimal design versus that of solving the problem with randomly generated designs with the same number of sensors. Note that the A-optimal sensor placement outperforms the random designs.

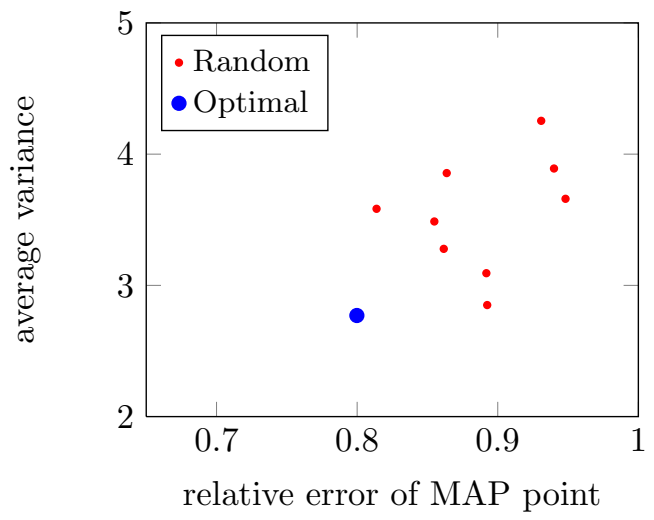

FIG. 7.4. Relative error of the MAP point versus average posterior variance for random designs (red dots) and for the optimal design (blue dot). The results corresponds to designs with 22 sensors.

8. Conclusions and remarks. We have developed a scalable method for computing A-optimal experimental designs for infinite-dimensional Bayesian nonlinear inverse problems governed by PDEs. By scalable, we mean that the cost (measured in forward-like PDE solves) of solving the OED problem is independent of the param- 
eter and sensor dimensions. The OED formulation results in a bilevel optimization problem that features an inverse problem as the inner optimization problem, and additional forward-like PDEs representing the action of the inverse Hessian of the inverse problem as constraints for the outer optimization problem. We specialize this OED formulation to the problem of determining the sensor placement that optimally infers the coefficient of an elliptic PDE in the sense that the uncertainty in the recovered coefficient is minimized over a set of prior model samples. For the resulting PDEconstrained OED problem, we derive adjoint-based expressions for the gradient, which enables use of efficient gradient-based optimization algorithms. Computing the gradient of the OED objective function requires differentiating expressions involving the Hessian, which requires third derivatives of the parameter-to-observable map. These are made tractable via a variational formulation of the OED problem. Numerical studies of the performance of our OED method for the inference of the log permeability field in a porous media flow problem indicate that the computational cost of computing an A-optimal experimental design, measured in the number of forward-like PDE solves, is insensitive to the dimension of the discretized parameter field and to the sensor dimension.

A potential limitation of our method is defining the OED objective in terms of a Gaussian approximation to the posterior distribution of the parameter field. However, as mentioned in the introduction, a Gaussian provides a good approximation to the posterior in cases where a linear approximation to the parameter-to-observable map over the set of parameters with significant posterior probability is sufficiently accurate. Relaxing the Gaussian approximation of the posterior for large-scale Bayesian inverse problems with expensive-to-evaluate parameter-to-observable maps is extremely challenging. The fact that the Bayesian inverse problem is merely an inner problem for computing OEDs compounds these challenges.

A related consideration is the influence of the prior on the OED obtained from our formulation. In cases where one has limited prior information, samples from the prior may have rather different features. Since data computed from these vastly different prior samples are used as "training data" in our OED formulation, the resulting design might be suboptimal for the "truth" parameter as we are searching for an A-optimal design that accommodates a wide range of data. In such cases, an effective strategy could be an iterative process: namely, one conducts initial field experiments and obtains a Bayesian update, which better constrains the uncertain parameter field. This field is then used as prior in the computation of an OED, whose target is to collect additional experimental data.

Another limitation of our approach is that our sparsification strategy provides only indirect control on the number of sensors in the optimal configuration. In practice, solving multiple OED problems may be required to determine an appropriate penalty parameter experimentally. This, however, is the price we pay to render an otherwise combinatorial sensor placement problem computationally tractable.

Computing optimal experimental designs still requires a large number of forward (or adjoint or incremental) PDE solves. However, as discussed in Section 5.5, a number of systems characterized by the same Hessian operator must be solved at each OED step, which suggests that using low rank Hessian approximations as discussed in $[1,7,15]$ can mitigate this computational cost. Moreover, our OED method contains important coarse-grained parallelism: the inverse problems corresponding to each data sample can be solved independently.

In future work, we intend to study the sensitivity of the optimal sensor placement 
to the number of data samples in the OED problem. The data samples are generated by sampling the prior model; their number is dictated by the need to solve an additional inverse problem for each sample at each OED iteration. For this reason, the numerical experiments in this paper have been limited to a small number of data samples. However, we speculate that increasing the number of data samples leads to diminishing returns, since the goal is not to fully sample the prior, but to determine optimal sensor locations, and we expect that they will be sensitive to only a limited number of directions in the parameter space. Thus, an interesting extension of this work is to determine how many data samples are needed.

Appendix A. An infinite-dimensional trace estimator. Let $\mu_{\delta}=\mathcal{N}\left(0, \mathcal{C}_{\delta}\right)$ and $\tilde{\mu}_{\delta}=\mathcal{N}\left(0, \mathcal{A}^{1 / 2} \mathcal{C}_{\delta} \mathcal{A}^{1 / 2}\right)$ with $\mathcal{A}$ and $\mathcal{C}_{\delta}$ as in the paragraph preceding (2.7); the final equality in (2.7) follows by noting that

$$
\int_{\mathscr{H}}\langle z, \mathcal{A} z\rangle \mu_{\delta}(d z)=\int_{\mathscr{H}}\left\|\mathcal{A}^{1 / 2} z\right\|^{2} \mu_{\delta}(d z)=\int_{\mathscr{H}}\|y\|^{2} \tilde{\mu}_{\delta}(d y)=\operatorname{tr}\left(\mathcal{A}^{1 / 2} \mathcal{C}_{\delta} \mathcal{A}^{1 / 2}\right)=\operatorname{tr}\left(\mathcal{A} \mathcal{C}_{\delta}\right) .
$$

The following result justifies taking the limit as we let $\delta \rightarrow 0$.

Proposition A.1. Let $\mathcal{D}$ be a bounded domain with Lipschitz boundary and consider the operator $\mathcal{C}_{\delta}=(-\delta \Delta+I)^{-2}$ defined on $L^{2}(\mathcal{D})$, where $\delta$ is a positive real and $\Delta$ is the Laplacian operator on $\mathcal{D}$ with the natural boundary condition. Suppose $\mathcal{A}$ is a positive self-adjoint trace-class operator on $L^{2}(\mathcal{D})$. Then,

$$
\lim _{\delta \rightarrow 0} \operatorname{tr}(\mathcal{A C} \delta)=\operatorname{tr}(\mathcal{A})
$$

Proof. Let us consider the difference, $\operatorname{tr}(\mathcal{A})-\operatorname{tr}\left(\mathcal{A C}_{\delta}\right)=\operatorname{tr}\left(\mathcal{A}\left(I-\mathcal{C}_{\delta}\right)\right)$. Denote by $\left\{e_{i}\right\}_{i=1}^{\infty}$ the eigenvectors of $I-\mathcal{C}_{\delta}$ (independent of $\delta$ ) and by $\lambda_{i}^{\delta}$ the respective eigenvalues. By the definition of the operator $\mathcal{C}_{\delta}$ we have $\lambda_{i}^{\delta}=1-1 /\left(1+\delta \nu_{i}\right)^{2}$ where $\nu_{i}$ are the (unbounded) eigenvalues of $-\Delta$. Using the fact that $0 \leq \nu_{i} \rightarrow \infty$, we know $0 \leq \lambda_{i}^{\delta}<1$ for all $\delta>0$. Next, we note

$$
\operatorname{tr}\left(\mathcal{A}\left(I-\mathcal{C}_{\delta}\right)\right)=\sum_{i=1}^{\infty}\left\langle e_{i}, \mathcal{A}\left(I-\mathcal{C}_{\delta}\right) e_{i}\right\rangle=\sum_{i=1}^{\infty} \lambda_{i}^{\delta}\left\langle e_{i}, \mathcal{A} e_{i}\right\rangle<\infty
$$

Let $\varepsilon>0$ be fixed but arbitrary and note that we can fix $N_{0} \in \mathbb{N}$ such that, $\sum_{i=N_{0}+1}^{\infty} \lambda_{i}^{\delta}\left\langle e_{i}, \mathcal{A} e_{i}\right\rangle \leq \sum_{i=N_{0}+1}^{\infty}\left\langle e_{i}, \mathcal{A} e_{i}\right\rangle<\varepsilon / 2$. Also, we can choose $\delta>0$ sufficiently small so that, $\sum_{i=1}^{N_{0}} \lambda_{i}^{\delta}\left\langle e_{i}, \mathcal{A} e_{i}\right\rangle \leq\|A\| \sum_{i=1}^{N_{0}} \lambda_{i}^{\delta}<\varepsilon / 2$, and hence the assertion of the proposition follows.

Appendix B. Gradient derivation of OED objective function $\hat{\Psi}$. Here, we summarize the derivation of the gradient of the OED objective function presented in (5.7). To derive the expression for the gradient, we employ a formal Lagrangian approach [35], which uses a Lagrangian function composed of the objective function (5.6a) with the PDE constraints (5.6b)-(5.6f) enforced through Lagrange multiplier functions. This Lagrangian function $\mathscr{L}^{\circ}$ for the OED problem is given by:

$$
\begin{aligned}
& \mathscr{L}^{\mathrm{O}}\left(\boldsymbol{w},\left\{u_{i}\right\},\left\{m_{i}\right\},\left\{p_{i}\right\},\left\{v_{i k}\right\},\left\{q_{i k}\right\},\left\{y_{i k}\right\},\left\{u_{i}^{*}\right\},\left\{m_{i}^{*}\right\},\left\{p_{i}^{*}\right\},\left\{v_{i k}^{*}\right\},\left\{q_{i k}^{*}\right\},\left\{y_{i k}^{*}\right\}\right) \\
& =\frac{1}{n_{\mathrm{d}} n_{\mathrm{tr}}} \sum_{i=1}^{n_{\mathrm{d}}} \sum_{k=1}^{n_{\mathrm{tr}}}\left\langle z_{k}, y_{i k}\right\rangle
\end{aligned}
$$




$$
\begin{aligned}
& +\sum_{i=1}^{n_{\mathrm{d}}}\left[\left\langle e^{m_{i}} \nabla u_{i}, \nabla u_{i}^{*}\right\rangle-\left\langle f, u_{i}^{*}\right\rangle-\left\langle h, u_{i}^{*}\right\rangle_{\Gamma_{N}}\right] \\
& +\sum_{i=1}^{n_{\mathrm{d}}}\left[\left\langle e^{m_{i}} \nabla p_{i}, \nabla p_{i}^{*}\right\rangle+\left\langle\mathcal{B}^{*} \mathbf{W}_{\sigma}\left(\mathcal{B} u_{i}-\mathbf{d}_{i}\right), p_{i}^{*}\right\rangle\right] \\
& +\sum_{i=1}^{n_{\mathrm{d}}}\left[\left\langle m_{i}-m_{\mathrm{pr}}, m_{i}^{*}\right\rangle_{\mathscr{E}}+\left\langle m_{i}^{*} e^{m_{i}} \nabla u_{i}, \nabla p_{i}\right\rangle\right] \\
& +\sum_{i=1}^{n_{\mathrm{d}}} \sum_{k=1}^{n_{\mathrm{tr}}}\left[\left\langle e^{m_{i}} \nabla v_{i k}, \nabla v_{i k}^{*}\right\rangle+\left\langle y_{i k} e^{m_{i}} \nabla u_{i}, \nabla v_{i k}^{*}\right\rangle\right] \\
& +\sum_{i=1}^{n_{\mathrm{d}}} \sum_{k=1}^{n_{\mathrm{tr}}}\left[\left\langle e^{m_{i}} \nabla q_{i k}, \nabla q_{i k}^{*}\right\rangle+\left\langle y_{i k} e^{m_{i}} \nabla p_{i}, \nabla q_{i k}^{*}\right\rangle+\left\langle\mathcal{B}^{*} \mathbf{W}_{\sigma} \mathcal{B} v_{i k}, q_{i k}^{*}\right\rangle\right] \\
& +\sum_{i=1}^{n_{\mathrm{d}}} \sum_{k=1}^{n_{\mathrm{tr}}}\left[\left\langle y_{i k}^{*} e^{m_{i}} \nabla v_{i k}, \nabla p_{i}\right\rangle+\left\langle y_{i k}^{*}, y_{i k}\right\rangle_{\mathscr{E}}+\left\langle y_{i k}^{*} e^{m_{i}} \nabla u_{i}, \nabla q_{i k}\right\rangle\right. \\
& \left.\quad+\left\langle y_{i k}^{*} y_{i k} e^{m_{i}} \nabla u_{i}, \nabla p_{i}\right\rangle-\left\langle z_{k}, y_{i k}^{*}\right\rangle\right] .
\end{aligned}
$$

The variables $\left(u_{i}, m_{i}, p_{i}\right) \in \mathscr{V}_{g} \times \mathscr{E} \times \mathscr{V}_{0}$, for $i \in\left\{1, \ldots, n_{\mathrm{d}}\right\}$, and $\left(v_{i k}, q_{i k}, y_{i k}\right) \in$ $\mathscr{V}_{0} \times \mathscr{V}_{0} \times \mathscr{E}$, with $(i, k) \in\left\{1, \ldots, n_{\mathrm{d}}\right\} \times\left\{1, \ldots, n_{\mathrm{tr}}\right\}$ are the OED state variables. The OED adjoint variables $u_{i}^{*}, m_{i}^{*}, p_{i}^{*}, v_{i k}^{*}, q_{i k}^{*}$, and $y_{i k}^{*}$ belong to the test function spaces corresponding to their state counterparts.

The gradient for (5.6a) is given by the derivative of $\mathscr{L}^{\circ}$ with respect to the weight vector $\boldsymbol{w}$, provided that variations of $\mathscr{L}^{\circ}$ with respect to the OED state and adjoint variables vanish. The weight vector enters the Lagrangian through the weight matrix $\mathbf{W}_{\sigma}=\sum_{j=1}^{n_{s}} w_{j} \mathbf{E}_{j}$, where $\mathbf{E}_{j}=\sigma_{j}^{-2} \boldsymbol{e}_{j} \boldsymbol{e}_{j}^{T}$. (Here $\boldsymbol{e}_{j}$ denotes the $j$ th standard basis vector in $\mathbb{R}^{n_{s}}$.) Using this notation, it is straightforward to compute derivatives of the Lagrangian function with respect to $w_{j}$, the $j$ th component of the weight vector $w$ :

$$
\mathscr{L}_{w_{j}}^{\mathrm{O}}=\sum_{i=1}^{n_{\mathrm{d}}}\left\langle\mathcal{B}^{*} \mathbf{E}_{j}\left(\mathcal{B} u_{i}-\mathbf{d}_{i}\right), p_{i}^{*}\right\rangle+\sum_{i=1}^{n_{\mathrm{d}}} \sum_{k=1}^{n_{\text {tr }}}\left\langle\mathcal{B}^{*} \mathbf{E}_{j} \mathcal{B} v_{i k}, q_{i k}^{*}\right\rangle, \quad \text { for } j=1, \ldots, n_{s} .
$$

Recalling the definition of $\mathbf{E}_{j}$ and using a vector form for the gradient, we obtain

$$
\hat{\Psi}^{\prime}=\sum_{i=1}^{n_{\mathrm{d}}} \boldsymbol{\Gamma}_{\text {noise }}^{-1}\left(\mathcal{B} u_{i}-\mathbf{d}_{i}\right) \odot \mathcal{B} p_{i}^{*}+\sum_{i=1}^{n_{\mathrm{d}}} \sum_{k=1}^{n_{\text {tr }}} \boldsymbol{\Gamma}_{\text {noise }}^{-1} \mathcal{B} v_{i k} \odot \mathcal{B} q_{i k}^{*},
$$

provided appropriate state and adjoint equations are satisfied. These equations are computed next.

Requiring that variations of $\mathscr{L}^{\circ}$ with respect to the OED adjoint variables vanish, we recover the OED state equations (5.6b)-(5.6f). The variables $p_{i k}^{*}$ and $q_{i k}^{*}$ are defined through adjoint equations, obtained by requiring that variations of $\mathscr{L}^{\circ}$ with respect to the OED state variables vanish. That is, for each $i \in\left\{1, \ldots, n_{\mathrm{d}}\right\}$ and $k \in\left\{1, \ldots, n_{\mathrm{tr}}\right\}$,

$$
\begin{aligned}
\mathscr{L}_{v_{i k}}^{\mathrm{O}}[\tilde{v}]= & \left\langle\mathcal{B}^{*} \mathbf{W}_{\sigma} \mathcal{B} \tilde{v}, q_{i k}^{*}\right\rangle+\left\langle y_{i k}^{*} e^{m_{i}} \nabla \tilde{v}, \nabla p_{i}\right\rangle+\left\langle e^{m_{i}} \nabla \tilde{v}, \nabla v_{i k}^{*}\right\rangle=0, \\
\mathscr{L}_{q_{i k}}^{\mathrm{O}}[\tilde{q}]= & \left\langle e^{m_{i}} \nabla \tilde{q}, \nabla q_{i k}^{*}\right\rangle+\left\langle y_{i k}^{*} e^{m_{i}} \nabla u_{i}, \nabla \tilde{q}\right\rangle=0, \\
\mathscr{L}_{y_{i k}}^{\mathrm{O}}[\tilde{y}]= & \left\langle\tilde{y} e^{m_{i}} \nabla p_{i}, \nabla q_{i k}^{*}\right\rangle+\left\langle y_{i k}^{*}, \tilde{y}\right\rangle_{\mathscr{E}}+\left\langle\tilde{y} y_{i k}^{*} e^{m_{i}} \nabla u_{i}, \nabla p_{i}\right\rangle+\left\langle\tilde{y} e^{m_{i}} \nabla u_{i}, \nabla v_{i k}^{*}\right\rangle \\
& +\frac{1}{n_{\mathrm{d}} n_{\mathrm{tr}}}\left\langle z_{k}, \tilde{y}\right\rangle=0,
\end{aligned}
$$




$$
\begin{aligned}
\mathscr{L}_{u_{i}}^{\mathrm{O}}[\tilde{u}]= & \left\langle\mathcal{B}^{*} \mathbf{W}_{\sigma} \mathcal{B} \tilde{u}, p_{i}^{*}\right\rangle+\left\langle m_{i}^{*} e^{m_{i}} \nabla \tilde{u}, \nabla p_{i}\right\rangle+\left\langle e^{m_{i}} \nabla \tilde{u}, \nabla u_{i}^{*}\right\rangle-\left\langle b_{i}^{(1)}, \tilde{u}\right\rangle=0, \\
\mathscr{L}_{m_{i}}^{\mathrm{O}}[\tilde{m}]= & \left\langle\tilde{m} e^{m_{i}} \nabla p_{i}, \nabla p_{i}^{*}\right\rangle+\left\langle m_{i}^{*}, \tilde{m}\right\rangle_{\mathscr{E}}+\left\langle\tilde{m} m_{i}^{*} e^{m_{i}} \nabla u_{i}, \nabla p_{i}\right\rangle \\
& +\left\langle\tilde{m} e^{m_{i}} \nabla u_{i}, \nabla u_{i}^{*}\right\rangle-\left\langle b_{i}^{(2)}, \tilde{m}\right\rangle=0, \\
\mathscr{L}_{p_{i}}^{\mathrm{O}}[\tilde{p}]= & \left\langle e^{m_{i}} \nabla \tilde{p}, \nabla p_{i}^{*}\right\rangle+\left\langle m_{i}^{*} e^{m_{i}} \nabla u_{i}, \nabla \tilde{p}\right\rangle-\left\langle b_{i}^{(3)}, \tilde{p}\right\rangle=0,
\end{aligned}
$$

for all $(\tilde{v}, \tilde{q}, \tilde{y}, \tilde{u}, \tilde{m}, \tilde{p}) \in \mathscr{V}_{0} \times \mathscr{V}_{0} \times \mathscr{E} \times \mathscr{V}_{0} \times \mathscr{E} \times \mathscr{V}_{0}$. Here, $b_{i}^{(1)}, b_{i}^{(2)}$, and $b_{i}^{(3)}$ are

$$
\begin{aligned}
\left\langle b_{i}^{(1)}, \tilde{u}\right\rangle=- & \sum_{k=1}^{n_{\mathrm{tr}}}\left[\left\langle y_{i k} e^{m_{i}} \nabla \tilde{u}, \nabla v_{i k}^{*}\right\rangle+\left\langle y_{i k}^{*} e^{m_{i}} \nabla \tilde{u}, \nabla q_{i k}\right\rangle+\left\langle y_{i k}^{*} y_{i k} e^{m_{i}} \nabla \tilde{u}, \nabla p_{i}\right\rangle\right], \\
\left\langle b_{i}^{(2)}, \tilde{m}\right\rangle=-\sum_{k=1}^{n_{\mathrm{tr}}}\left[\left\langle\tilde{m} e^{m_{i}} \nabla v_{i k}, \nabla v_{i k}^{*}\right\rangle+\left\langle\tilde{m} e^{m_{i}} \nabla q_{i k}, \nabla q_{i k}^{*}\right\rangle+\left\langle\tilde{m} y_{i k} e^{m_{i}} \nabla u_{i}, \nabla v_{i k}^{*}\right\rangle\right. & +\left\langle\tilde{m} y_{i k} e^{m_{i}} \nabla p_{i}, \nabla q_{i k}^{*}\right\rangle+\left\langle\tilde{m} y_{i k}^{*} e^{m_{i}} \nabla v_{i k}, \nabla p_{i}\right\rangle+\left\langle\tilde{m} y_{i k}^{*} e^{m_{i}} \nabla u_{i}, \nabla q_{i k}\right\rangle \\
\quad & \left.\quad\left\langle\tilde{m} y_{i k}^{*} y_{i k} e^{m_{i}} \nabla u_{i}, \nabla p_{i}\right\rangle\right], \\
\left\langle b_{i}^{(3)}, \tilde{p}\right\rangle=- & \sum_{k=1}^{n_{\mathrm{tr}}}\left[\left\langle y_{i k} e^{m_{i}} \nabla \tilde{p}, \nabla q_{i k}^{*}\right\rangle+\left\langle y_{i k}^{*} e^{m_{i}} \nabla v_{i k}, \nabla \tilde{p}\right\rangle+\left\langle y_{i k}^{*} y_{i k} e^{m_{i}} \nabla u_{i}, \nabla \tilde{p}\right\rangle\right] .
\end{aligned}
$$

Upon inspecting the OED adjoint equations (B.2)-(B.7) and comparing them to the system of equations (5.6b)-(5.6f), we notice that the OED adjoint equations inherit structure from the OED state equations. Specifically, notice that after rearranging and identifying terms, the system (B.2)-(B.4) for $\left(q_{i k}^{*}, v_{i k}^{*}, y_{i k}^{*}\right)$ is the same as the system (5.6g)-(5.6f), except for the right hand sides, which coincide up to a constant. This reveals the following relations:

$$
q_{i k}^{*}=-\frac{1}{n_{\mathrm{d}} n_{\mathrm{tr}}} v_{i k}, \quad y_{i k}^{*}=-\frac{1}{n_{\mathrm{d}} n_{\mathrm{tr}}} y_{i k}, \quad v_{i k}^{*}=-\frac{1}{n_{\mathrm{d}} n_{\mathrm{tr}}} q_{i k},
$$

for $i \in\left\{1, \ldots, n_{\mathrm{d}}\right\}$ and $k \in\left\{1, \ldots, n_{\mathrm{tr}}\right\}$. Thus, the OED adjoint variables $q_{i k}^{*}, y_{i k}^{*}$, and $v_{i k}^{*}$ can be eliminated from the system and the right hand sides $b_{i k}^{(1)}, b_{i k}^{(2)}, b_{i k}^{(3)}$ defined in (B.8) simplify, and result in (5.9).

Appendix C. Discretization and computational details. We use a finiteelement discretization of the parameter field and the state and adjoint variables, and we denote by boldfaced letters the discretized versions of the variables and operators appearing in the expressions. Next, we describe the numerical computation of the OED objective function in (5.6a) and of its gradient, where we again consider that $\gamma=0$. The discrete OED function is

$$
\hat{\Psi}_{h}(\boldsymbol{w})=\frac{1}{n_{\mathrm{d}} n_{\mathrm{tr}}} \sum_{i=1}^{n_{\mathrm{d}}} \sum_{k=1}^{n_{\mathrm{tr}}}\left\langle\boldsymbol{z}_{k}, \boldsymbol{y}_{i k}\right\rangle_{\mathrm{M}}
$$

Note that, to discretize the infinite-dimensional Hilbert space, we use a mass-weighted inner product in (C.1). This is necessary since the finite-dimensional inference parameters are the coefficients of the finite element approximation, and helps to ensure that the discrete problems are appropriate discretizations of the infinite-dimensional problem. We rely on a Gaussian trace estimator and let $\boldsymbol{z}_{k}=\mathbf{M}^{-1 / 2} \boldsymbol{\nu}_{k}, k=1, \ldots, n_{\mathrm{tr}}$, 
where $\boldsymbol{\nu}_{k}$ are draws from $\mathcal{N}(\mathbf{0}, \boldsymbol{I})$. See [1] for a justification of the form of the massweighted trace estimator and also an efficient procedure for computing the application of $\mathbf{M}^{-1 / 2}$ to a vector.

For a given design $\boldsymbol{w}$ and data samples $\mathbf{d}_{i}, i \in\left\{1, \ldots, n_{\mathrm{d}}\right\}$, we solve the inner optimization problem (5.6b)-(5.6d) for the MAP point $\boldsymbol{m}_{i}=\boldsymbol{m}_{\mathrm{MAP}}\left(\boldsymbol{w} ; \mathbf{d}_{i}\right)$; we also evaluate the state $\boldsymbol{u}_{i}$ and adjoint $\boldsymbol{p}_{i}$ variables (for the inner optimization) at the MAP point. Next, we need to solve for $\boldsymbol{y}_{i k}$ and the variables $\boldsymbol{v}_{i k}$ and $\boldsymbol{q}_{i k}$ in (5.6g)-(5.6f). This is accomplished by solving a linear system of the following block form

$$
\left[\begin{array}{ccc}
\mathbf{D} & \mathbf{S}^{T} & \mathbf{A}^{T} \\
\mathbf{S} & \mathbf{Q} & \mathbf{C}^{T} \\
\mathbf{A} & \mathbf{C} & \mathbf{0}
\end{array}\right]\left[\begin{array}{l}
\boldsymbol{v}_{i k} \\
\boldsymbol{y}_{i k} \\
\boldsymbol{q}_{i k}
\end{array}\right]=\left[\begin{array}{c}
\mathbf{0} \\
\boldsymbol{z}_{k} \\
\mathbf{0}
\end{array}\right]
$$

In the above system, $\mathbf{D}=\mathbf{B}^{T} \mathbf{W}_{\sigma} \mathbf{B}$, where $\mathbf{B}$ is the discretization of the observation operator $\mathcal{B}$. The remaining blocks in the system are discretizations of the differential operators appearing in (5.6e)-(5.6g), evaluated at $\left(\boldsymbol{u}_{i}, \boldsymbol{m}_{i}, \boldsymbol{p}_{i}\right)$; we refer to [28] for more details on the discretization of the Hessian system for an inverse coefficient problem with an elliptic PDE. To solve the system (C.2), we first block eliminate $\boldsymbol{v}_{i k}$ and $\boldsymbol{q}_{i k}$, namely

$$
\boldsymbol{v}_{i k}=-\mathbf{A}^{-1} \mathbf{C} \boldsymbol{y}_{i k}, \quad \boldsymbol{q}_{i k}=-\mathbf{A}^{-T}\left(\mathbf{D} \boldsymbol{v}_{i k}+\mathbf{S}^{T} \boldsymbol{y}_{i k}\right),
$$

for $i \in\left\{1, \ldots, n_{\mathrm{d}}\right\}$ and $k \in\left\{1, \ldots, n_{\mathrm{tr}}\right\}$, and solve $\mathbf{H} \boldsymbol{y}_{i k}=\boldsymbol{z}_{k}$ with

$$
\mathbf{H}=\mathbf{C}^{T} \mathbf{A}^{-T}\left(\mathbf{D A}{ }^{-1} \mathbf{C}-\mathbf{S}^{T}\right)-\mathbf{S A}^{-1} \mathbf{C}+\mathbf{Q} .
$$

Once $\boldsymbol{y}_{i k}$ is available for $i \in\left\{1, \ldots, n_{\mathrm{d}}\right\}$ and $k \in\left\{1, \ldots, n_{\mathrm{tr}}\right\}$, we can compute the OED objective function (C.1).

To compute the gradient we also need the OED adjoint variables $\boldsymbol{p}_{i}^{*}, i=1, \ldots, n_{\mathrm{d}}$, which are computed by solving a linear system similar to (C.2), for $\left(\boldsymbol{p}_{i}^{*}, \boldsymbol{m}_{i}^{*}, \boldsymbol{u}_{i}^{*}\right)$, where the blocks in the system right hand side are replaced by $\boldsymbol{b}_{i}^{(1)}, \boldsymbol{b}_{i}^{(2)}$, and $\boldsymbol{b}_{i}^{(3)}$ which are discretizations of the expressions in (5.9). Thus, we solve $\mathbf{H} \boldsymbol{m}_{i}^{*}=\overline{\boldsymbol{b}}_{i}$, where $\mathbf{H}$ is as in (C.3), and $\overline{\boldsymbol{b}}_{i}$ is given by

$$
\overline{\boldsymbol{b}}_{i}=\boldsymbol{b}_{i}^{(2)}-\mathbf{C}^{T} \mathbf{A}^{-T} \boldsymbol{b}_{i}^{(1)}-\mathbf{S} \mathbf{A}^{-1} \boldsymbol{b}_{i}^{(3)}+\mathbf{C}^{T} \mathbf{A}^{-T} \mathbf{D} \mathbf{A}^{-1} \boldsymbol{b}_{i}^{(3)},
$$

for $i \in\left\{1, \ldots, n_{\mathrm{d}}\right\}$. Next, we solve for $\boldsymbol{p}_{i}^{*}$,

$$
\boldsymbol{p}_{i}^{*}=\mathbf{A}^{-1}\left(\boldsymbol{b}_{i}^{(3)}-\mathbf{C m}_{i}^{*}\right), \quad i \in\left\{1, \ldots, n_{\mathrm{d}}\right\} .
$$

Subsequently, we have all the quantities required in the expression for the (discretized) gradient:

$$
\nabla \hat{\Psi}_{h}(\boldsymbol{w})=\sum_{i=1}^{n_{\mathrm{d}}} \boldsymbol{\Gamma}_{\text {noise }}^{-1}\left(\mathbf{B} \boldsymbol{u}_{i}-\mathbf{d}_{i}\right) \odot \mathbf{B} \boldsymbol{p}_{i}^{*}-\frac{1}{n_{\mathrm{d}} n_{\mathrm{tr}}} \sum_{i=1}^{n_{\mathrm{d}}} \sum_{k=1}^{n_{\mathrm{tr}}} \boldsymbol{\Gamma}_{\text {noise }}^{-1} \mathbf{B} \boldsymbol{v}_{i k} \odot \mathbf{B} \boldsymbol{v}_{i k} .
$$

\section{REFERENCES}

[1] A. Alexanderian, N. Petra, G. Stadler, and O. Ghattas, A-optimal design of experiments for infinite-dimensional Bayesian linear inverse problems with regularized $\ell_{0}$ sparsification, SIAM Journal on Scientific Computing, 36 (2014), pp. A2122-A2148. 
[2] A. C. Atkinson and A. N. Donev, Optimum Experimental Designs, Oxford, 1992.

[3] H. Avron And S. Toledo, Randomized algorithms for estimating the trace of an implicit symmetric positive semi-definite matrix, Journal of the ACM (JACM), 58 (2011), p. 17.

[4] I. Bauer, H. G. Bock, S. Körkel, And J. P. Schlöder, Numerical methods for optimum experimental design in DAE systems, Journal of Computational and Applied Mathematics, 120 (2000), pp. 1-25. SQP-based direct discretization methods for practical optimal control problems.

[5] H. G. Bock, S. KÖRKEL, AND J. P. SChlÖDER, Parameter estimation and optimum experimental design for differential equation models, in Model Based Parameter Estimation, H. G. Bock, T. Carraro, W. Jäger, S. Körkel, R. Rannacher, and J. P. Schlöder, eds., vol. 4 of Contributions in Mathematical and Computational Sciences, Springer Berlin Heidelberg, 2013, pp. 1-30.

[6] A. Borzì And V. Schulz, Computational Optimization of Systems Governed by Partial Differential Equations, SIAM, 2012.

[7] T. Bui-Thanh, O. Ghattas, J. Martin, and G. Stadler, A computational framework for infinite-dimensional Bayesian inverse problems Part I: The linearized case, with application to global seismic inversion, SIAM Journal on Scientific Computing, 35 (2013), pp. A2494-A2523.

[8] S. L. Campbell, I. C. F. Ipsen, C. T. Kelley, C. D. Meyer, and Z. Q. Xue, Convergence estimates for solution of integral equations with GMRES, J. Integral Eqs. and Applications, 8 (1996), pp. 19-34.

[9] M. ChUng AND E. HABER, Experimental design for biological systems, SIAM Journal on Control and Optimization, 50 (2012), pp. 471-489.

[10] G. Da Prato, An Introduction to Infinite-dimensional Analysis, Universitext, Springer, 2006.

[11] M. Dashti, S. Harris, And A. StuART, Besov priors for Bayesian inverse problems, Inverse Problems and Imaging, 6 (2012), pp. 183-200.

[12] M. Dashti, K. J. Law, A. M. Stuart, and J. Voss, MAP estimators and their consistency in Bayesian nonparametric inverse problems, Inverse Problems, 29 (2013), p. 095017.

[13] M. Dashti And A. M. StuART, The Bayesian approach to inverse problems, in Handbook of Uncertainty Quantification, R. Ghanem, D. Higdon, and H. Owhadi, eds., Spinger, 2015.

[14] P. Deuflhard, Newton methods for nonlinear problems, vol. 35 of Springer Series in Computational Mathematics, Springer-Verlag, Berlin, 2004.

[15] H. P. Flath, L. C. Wilcox, V. Akçelik, J. Hill, B. van Bloemen Waanders, and O. GhatTAS, Fast algorithms for Bayesian uncertainty quantification in large-scale linear inverse problems based on low-rank partial Hessian approximations, SIAM Journal on Scientific Computing, 33 (2011), pp. 407-432.

[16] E. Haber, L. HoResh, AND L. Tenorio, Numerical methods for experimental design of largescale linear ill-posed inverse problems, Inverse Problems, 24 (2008), pp. 125-137.

[17] E. Haber, L. Horesh, and L. Tenorio, Numerical methods for the design of large-scale nonlinear discrete ill-posed inverse problems, Inverse Problems, 26 (2010), p. 025002.

[18] E. Haber, Z. Magnant, C. Lucero, and L. Tenorio, Numerical methods for A-optimal designs with a sparsity constraint for ill-posed inverse problems, Computational Optimization and Applications, (2012), pp. 1-22.

[19] L. Horesh, E. Haber, and L. Tenorio, Optimal Experimental Design for the Large-Scale Nonlinear Ill-Posed Problem of Impedance Imaging, Wiley, 2010, pp. 273-290.

[20] X. Huan And Y. M. Marzouk, Simulation-based optimal Bayesian experimental design for nonlinear systems, Journal of Computational Physics, 232 (2013), pp. 288-317.

[21] — Gradient-based stochastic optimization methods in Bayesian experimental design, International Journal for Uncertainty Quantification, 4 (2014), pp. 479-510.

[22] M. F. Hutchinson, A stochastic estimator of the trace of the influence matrix for Laplacian smoothing splines, Communications in Statistics-Simulation and Computation, 19 (1990), pp. $433-450$.

[23] S. Körkel, E. Kostina, H. G. Bock, And J. P. Schlöder, Numerical methods for optimal control problems in design of robust optimal experiments for nonlinear dynamic processes, Optimization Methods \& Software, 19 (2004), pp. 327-338. The First International Conference on Optimization Methods and Software. Part II.

[24] M. Lassas, E. Saksman, and S. Siltanen, Discretization invariant Bayesian inversion and Besov space priors, Inverse Problems and Imaging, 3 (2009), pp. 87-122.

[25] F. Lindgren, H. Rue, and J. Lindström, An explicit link between Gaussian fields and Gaussian Markov random fields: the stochastic partial differential equation approach, Journal of the Royal Statistical Society: Series B (Statistical Methodology), 73 (2011), pp. 423-498.

[26] Q. Long, M. Scavino, R. Tempone, and S. Wang, Fast estimation of expected informa- 
tion gains for Bayesian experimental designs based on Laplace approximations, Computer Methods in Applied Mechanics and Engineering, 259 (2013), pp. 24-39.

[27] A. PÁzman, Foundations of Optimum Experimental Design, D. Reidel Publishing Co., 1986.

[28] N. Petra and G. Stadler, Model variational inverse problems governed by partial differential equations, Tech. Rep. 11-05, The Institute for Computational Engineering and Sciences, The University of Texas at Austin, 2011.

[29] G. D. Prato and J. Zabczyk, Stochastic Equations in Infinite Dimensions, Cambidge University Press, 1992.

[30] F. Pukelsheim, Optimal Design of Experiments, John Wiley \& Sons, New-York, 1993.

[31] C. P. Robert and G. Casella, Monte Carlo Statistical Methods (Springer Texts in Statistics), Springer-Verlag New York, Inc., Secaucus, NJ, USA, 2005.

[32] F. Roosta-Khorasani And U. Ascher, Improved bounds on sample size for implicit matrix trace estimators. Preprint, 2013.

[33] A. M. Stuart, Inverse problems: A Bayesian perspective, Acta Numerica, 19 (2010), pp. 451559.

[34] A. TARantola, Inverse Problem Theory and Methods for Model Parameter Estimation, SIAM, Philadelphia, PA, 2005.

[35] F. Tröltzsch, Optimal Control of Partial Differential Equations: Theory, Methods and Applications, vol. 112 of Graduate Studies in Mathematics, American Mathematical Society, 2010.

[36] D. UCIŃSKI, Optimal measurement methods for distributed parameter system identification, CRC Press, Boca Raton, 2005.

[37] D. Williams, Probability with Martingales, Cambridge University Press, 1991. 